\title{
Aminolysis of $N$-tosylaziridines: an approach to asymmetric synthesis of symmetric and unsymmetric chiral sulfonamide ligands
}

\author{
Alakesh Bisai, B. A. Bhanu Prasad, and Vinod K. Singh* \\ Department of Chemistry, Indian Institute of Technology, Kanpur, India - 208016 \\ E-mail:vinodks@iitk.ac.in
}

Dedicated with respect to Professor Lutz Tietze on his $65^{\text {th }}$ birthday

\begin{abstract}
The ring-opening of $\mathrm{N}$-tosylaziridines with aliphatic amines can be efficiently catalyzed by lithium perchlorate to provide derivatives of the trans-1,2-diamine in high yields. The reaction was used in desymmetrization of several cyclic $N$-tosylaziridines using chiral amines. Using this strategy, an efficient synthesis was developed of chiral vicinal $C_{2}$ symmetric bis-(sulfonamides), unsymmetrical bis(sulfonamides) and other symmetric and unsymmetric ligands based on trans1,2-cyclohexanediamine.
\end{abstract}

Keywords: Aminolysis, bis(sulfonamide) ligands, trans-1,2-cyclohexanediamine, lithium perchlorate, $N$-tosylaziridines

\section{Introduction}

The development of methods for the synthesis new chiral ligands are an important aspect in the advancement of catalytic asymmetric synthesis because small changes in the donating ability of a ligand or the size of its substituents can have a dramatic effect on the catalyst efficiency and enantioselectivity. ${ }^{1-3} C_{2}$-Symmetric bis(sulfonamide) ligands of the type 1 (Figure 1) are electronically different and bind well to early transition metals ${ }^{4}$ and main-group elements. ${ }^{5}$ This type of ligand has been used in the asymmetric Diels-Alder reaction, ${ }^{5,6}$ the alkylation of aldehydes, ${ }^{7}$ the cyclopropanation of allylic alcohols ${ }^{8}$ and the amination of $N$-acyloxazolidones. ${ }^{9}$ The chemistry of these ligands has been very well studied in the field of asymmetric synthesis. However, not much work has been done using unsymmetrical bis(sulfonamide) ligands of type 2. ${ }^{10,11}$ This is mainly due to their non-availability and difficulty in the mono-sulfonylation of the 1,2-diamine such as 3. Palladium- catalyzed monoarylation of 1,2-diamine 3 has been developed, but the yields were not high. ${ }^{12}$ Walsh and coworkers have developed a very good procedure for the synthesis of unsymmetrical bis(sulfonamides) via an amino-sulfonamide 4 from the 
commercially available corresponding diamine. ${ }^{13 a, b}$ In this paper we describe full details of our work towards a general approach to this kind of chiral ligand, based on the aminolysis of aziridines. $^{13 \mathrm{c}}$

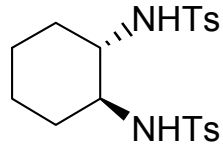

$(S, S)-1$

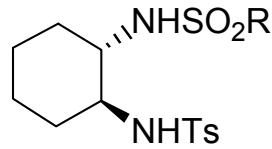

$\mathrm{R}=$ alkyl or aryl

$(S, S)-2$<smiles>N[C@@H]1CCCC[C@H]1N</smiles>

$(S, S)-\mathbf{3}$

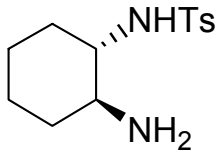

$(S, S)-\mathbf{4}$

Figure 1. Cyclohexane-based symmetrical and unsymmetrical chiral ligands.

\section{Results and Discussion}

It was envisaged that if $N$-tosylcyclohexyl aziridine were opened with a benzylamine in a diastereoselective manner, the product could be converted into an amino-sulfonamide after debenzylation. With this idea, several $N$-tosyl aziridines were synthesized using known procedures. ${ }^{14}$ Although ring-opening of such $\mathrm{N}$-activated aziridines with aromatic amines has been studied extensively using Lewis acids, ${ }^{15}$ little has been published on the opening with aliphatic amines. ${ }^{16}$ At the outset, 10 mol.\% of several Lewis acids such as $\mathrm{Cu}(\mathrm{OTf})_{2}(70 \%)$, $\mathrm{Zn}(\mathrm{OTf})_{2}(81 \%), \mathrm{Sn}(\mathrm{OTf})_{2}(78 \%), \mathrm{YbCl}_{3}(70 \%), \mathrm{ErCl}_{3}(68 \%)$, and $\mathrm{LiClO}_{4}(88 \%)$, were screened for opening of $N$-tosylcyclohexyl aziridine $(1 \mathrm{mmol})$ with benzylamine $(1.25 \mathrm{mmol})$ in $\mathrm{MeCN}$ at room temperature over more than $24 \mathrm{~h}$. From this study, it appeared that $\mathrm{LiClO}_{4}$ is more effective, as indicated by isolated yields. Long reaction times at room temperature prompted us to try the same reaction at reflux temperature using $\mathrm{LiClO}_{4}$ as a catalyst. To our delight, the reaction was complete in $4 \mathrm{~h}$ and the ring-opened product was obtained in 94\% yield (Table 1, entry 1). The trans- stereochemistry in the product was deduced from the coupling constants $(J=$ $10.5 \mathrm{~Hz}$ and $3.9 \mathrm{~Hz})$ of the signal at $2.29 \mathrm{ppm}(-\mathrm{CH}-\mathrm{NH}-)$ in the ${ }^{1} \mathrm{H}-\mathrm{NMR}$ spectrum. The ring opening reaction was also tried with other amines such as phenylethylamine, piperidine, morpholine, and $N$-ethoxycarbonyl piperazine. In all cases, high yields of product were obtained (Table 1, entries 2-5). The reaction was extended to a few other $N$-tosyl aziridines and the results are summarized in Table 1. Aziridines derived from cyclopentene and cyclohexa-1,4-diene gave the ring-opened products in good to excellent yields (Table 1, entries 6-9). An acyclic terminal aziridine gave products resulting from terminal attack only (Table 1, entries 10 and 11).

Once the methodology for cleavage of $N$-tosylaziridines with aliphatic amines, ${ }^{18}$ especially benzylamine, was established it was extended to chiral amines. Initially, $(R)-\alpha$ methylbenzylamine $(1.25 \mathrm{mmol})$ was used for desymmetrization of $N$-tosylcyclohexyl aziridine $(1 \mathrm{mmol})$ in the presence of $\mathrm{LiClO}_{4}(0.1 \mathrm{mmol})$ in $\mathrm{MeCN}$. The reaction was complete in $6 \mathrm{~h}$ at reflux temperature and the product was obtained in $94 \%$ yield as a separable mixture of diastereomers in a 1:1 ratio (Table 2, entry 1). The reaction was scaled up to $25 \mathrm{~g}$ scale without any problem. The $(S, S, R)-9$ diastereomer is more polar $\left(R_{\mathrm{f}} 0.28\right)$ than $(R, R, R)-9\left(R_{\mathrm{f}} 0.42\right)$. The 
absolute stereochemistry was established by X-ray analysis (vide infra). The ring opening reaction proceeded well with $(R)$-3,3-dimethyl-2-butylamine also, and a separable mixture of diastereomers (1:1 ratio) was obtained in $82 \%$ yield (Table 2, entry 2 ). In all cases the diastereomeric ratio was determined by ${ }^{1} \mathrm{H}-\mathrm{NMR}$ spectroscopy. The above reaction was extended to the tosylaziridine derived from cyclopentene also. Although good- to excellent yields of the ring-opened products were obtained, the diastereomers could not be separated by column chromatography. Another chiral amine, $(R)$-1-(3-methoxy phenyl)ethylamine, was also tried, but was no help (Table 2, entry 4). The mono tosylaziridine of cyclohexa-1,4-diene gave a similar result to that of cyclohexene, where diastereomers could be separated (Table 2, entry 6).

Table 1. Reaction of $N$-tosyl aziridines with aliphatic amines catalyzed by $\mathrm{LiClO}_{4}$ in $\mathrm{MeCN}$ at reflux temperature ${ }^{\mathrm{a}}$

\begin{tabular}{|c|c|c|c|c|}
\hline Entry & Aziridine & Products & & $\%$ Yield (time) ${ }^{b}$ \\
\hline 1. & & & $\mathbf{5 a}^{15 \mathrm{a}} ; \mathrm{R}^{1}=\mathrm{H}, \mathrm{R}^{2}=\mathrm{CH}_{2} \mathrm{Ph}$ & $94(4 \mathrm{~h})$ \\
\hline 2. & & & $\mathbf{5} \mathbf{b}^{16 b} ; \mathrm{R}^{1}=\mathrm{H}, \mathrm{R}^{2}=\mathrm{CH}_{2} \mathrm{CH}_{2} \mathrm{Ph}$ & $87(5 \mathrm{~h})$ \\
\hline 3. & & & $\mathbf{5 c} ; \mathrm{NR}^{1} \mathrm{R}^{2}=$ piperidino & $88(2 \mathrm{~h})$ \\
\hline 4. & & & 5d; $\mathrm{NR}^{1} \mathrm{R}^{2}=$ morpholino & $95(5 \mathrm{~h})$ \\
\hline 5. & & & $\begin{array}{l}\mathbf{5 e} ; \mathrm{NR}^{1} \mathrm{R}^{2}=N \text {-ethoxycarbonyl } \\
\text { pinerazine }\end{array}$ & $96(2 \mathrm{~h})$ \\
\hline 6. & & & $\mathbf{6 a}^{17} ; \mathrm{R}^{1}=\mathrm{H}, \mathrm{R}^{2}=\mathrm{CH}_{2} \mathrm{Ph}$ & $88(6 \mathrm{~h})$ \\
\hline 7. & & & $\mathbf{6 b} ; \mathrm{NR}^{1} \mathrm{R}^{2}=$ morpholino & $93(5 \mathrm{~h})$ \\
\hline 8. & & & $7 \mathbf{a} ; \mathrm{R}^{1}=\mathrm{H}, \mathrm{R}^{2}=\mathrm{CH}_{2} \mathrm{Ph}$ & $92(6 \mathrm{~h})$ \\
\hline 9. & & & $7 \mathbf{b} ; \mathrm{NR}^{1} \mathrm{R}^{2}=$ morpholino & $92(6 \mathrm{~h})$ \\
\hline 10. & & & & $89(4 \mathrm{~h})$ \\
\hline 11. & & & & $90(4 \mathrm{~h})$ \\
\hline
\end{tabular}

${ }^{\mathrm{a}}$ The ratio of $N$-tosylaziridine, aliphatic amine, and $\mathrm{LiClO}_{4}$ was 1.0:1.25:0.1. ${ }^{\mathrm{b}}$ Isolated yield after column chromatography over silica gel, using hexane and ethyl acetate as eluents.

In order to prove the absolute stereochemistry of the product $\mathbf{9}$, one of its diastereomers (the less polar) was methylated with MeI to prepare 15a whose crystal structure (Figure 2) 
proved it to be $(R, R, R)-9$. In order to show the versatility, several alkylated amines were synthesized (Scheme 1). Surprisingly, alkylation of $(R, R, R)-9$ with 2-methoxybenzyl bromide under identical conditions did not give the desired product. Instead, the reaction took place on the nitrogen to which the tosyl group was attached, thus giving 16 in $72 \%$ yield (Scheme 1). The structure was confirmed by an X-ray crystal structure determination (Figure 2). This result is strange, especially given the fact that the same reaction with benzyl bromide provided $\mathbf{1 5 f}$ where alkylation took place on the other nitrogen. The reason for this anomaly could be due to steric interaction between the methyl group on the chiral center and ortho-methoxy group on the benzyl bromide.

Table 2. Reaction of $N$-tosyl aziridines with chiral aliphatic amines catalyzed by $\mathrm{LiClO}_{4}$ in $\mathrm{MeCN}$ at reflux temperature

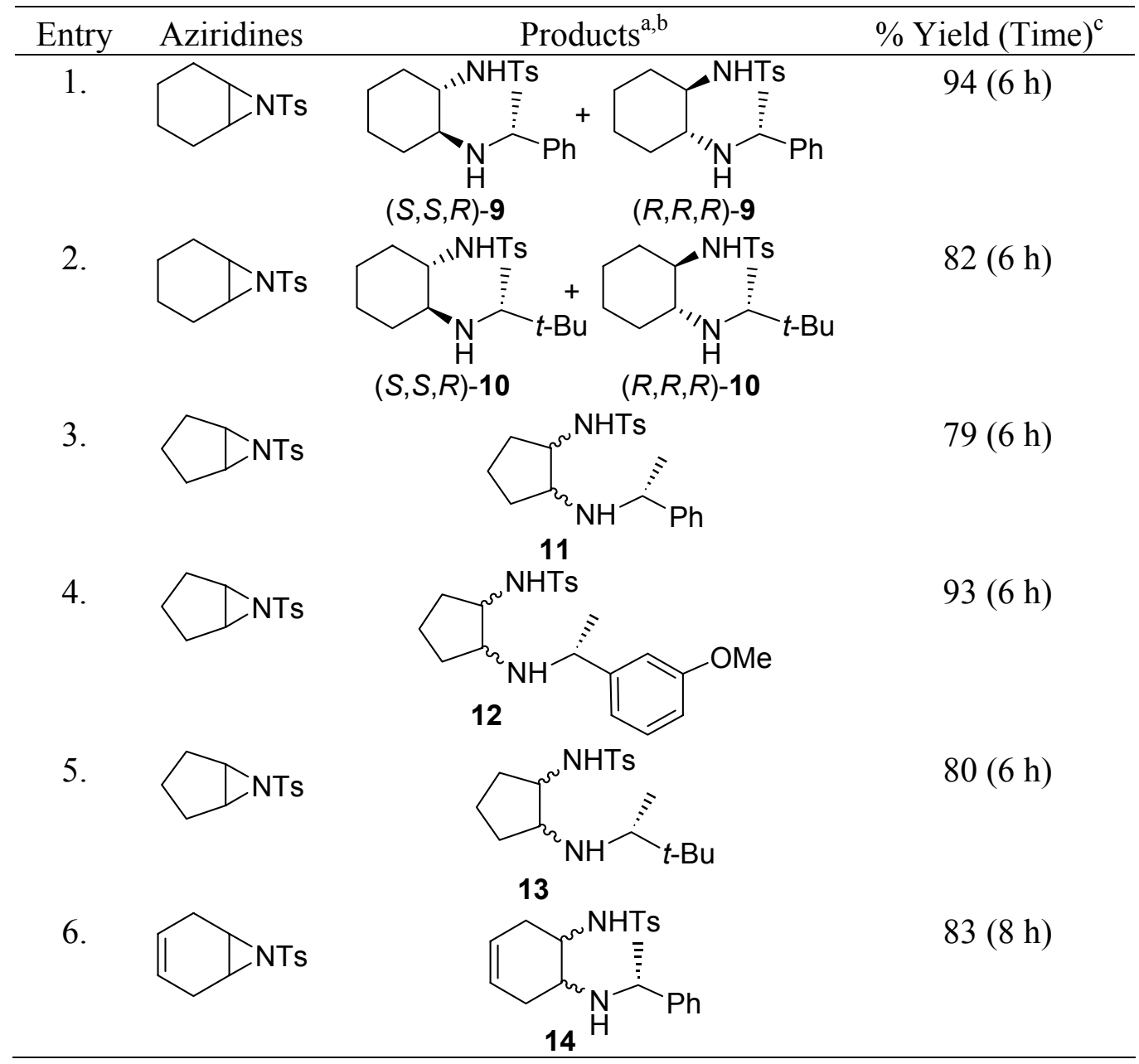

${ }^{a}$ The ratio of N-tosylaziridine, chiral amine, and $\mathrm{LiClO}_{4}$ was 1.0:1.25:0.1 and the products were obtained in 1:1 diastereomeric ratio. ${ }^{b}$ The diastereomeric ratio was determined by ${ }^{1} \mathrm{H}$ - NMR spectroscopy in all cases. ${ }^{\mathrm{c}}$ The diastereomers separated on TLC only for entries 1, 2, and 6. 


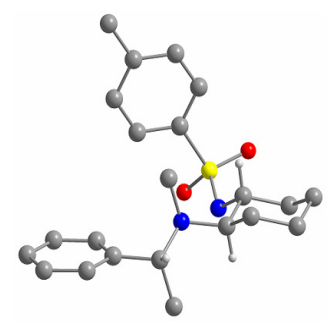

$(R, R, R)-15 \mathbf{a}$

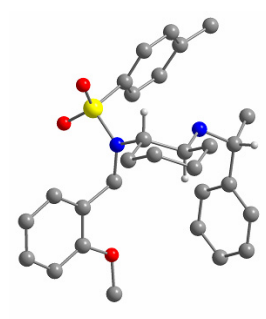

$(R, R, R)-16$

Figure 2. X-ray crystal structure of sulfonamides. ${ }^{19}$

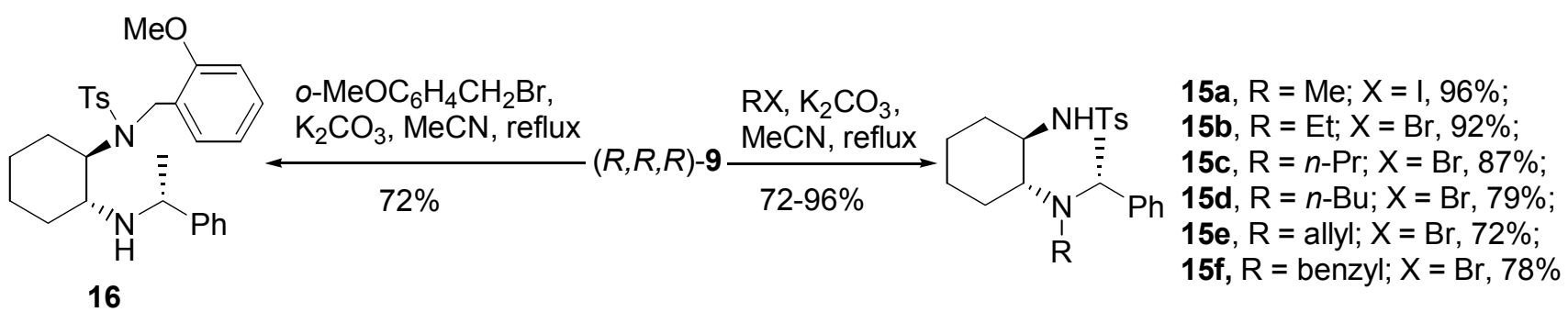

Scheme 1. Synthesis of sulfonamide ligands from an intermediate $(R, R, R)-9$.

After successful chromatographic separation of the enantiopure ring-opened product 9, we planned to synthesize several chiral ligands. Thus, the $(S, S, R)-9$ diastereomer was subjected to debenzylation $\left(\mathrm{Pd} / \mathrm{C}\right.$ and $\left.\mathrm{HCOONH}_{4}\right)$ to provide the amino-sulfonamide $(S, S)-\mathbf{4}$ in $91 \%$ yield. This is an important precursor for several chiral ligands. For example, $(S, S)$-4 was tosylated to provide the $C_{2}$ symmetric bis(sulfonamide) ligand $(S, S)$-1. Its treatment with various sulfonyl chlorides provided a series of enantiopure unsymmetrical vicinal bis(sulfonamide) ligands $(S, S)$ $\mathbf{2 a - h}$ in good to excellent yields. The amino-sulfonamide $(S, S)-\mathbf{4}$ compound was also converted into an important chiral ligand $17^{10 \mathrm{a}}$ in quantitative yield. ${ }^{13 \mathrm{a}}$

Pyridine-based bis(amide) is also well known ligand to bind well to transition metal complexes. ${ }^{20}$ These ligands are also important as far as enantioselective transformations are concerned. The aminosulfonamide $(S, S)-\mathbf{4}$ furnished the $C_{2}$-symmetric pyridyl bis(amide) bis(sulfonamide) ligand $(S, S)$-18 when treated with 0.5 equivalent of 2,6-pyridinedicarbonyl dichloride in dichloromethane in the presence of triethylamine. Similarly, $(S, S)$-19 was prepared using 3-picolinic acid (Scheme 3). ${ }^{13 \mathrm{~b}}$ We have synthesized L-Prolinamide based on trans-1,2diaminocyclohexane $(S, S, S)-21$ and $(R, R, S)$-21 by the coupling of aminosulfonamide $(S, S)-4$ with $N$-Boc- $L$-Proline followed by deprotection of the Boc group using $\mathrm{HCOOH}$. These chiral ligands would be useful in asymmetric organocatalytic aldol reactions. ${ }^{21}$ 


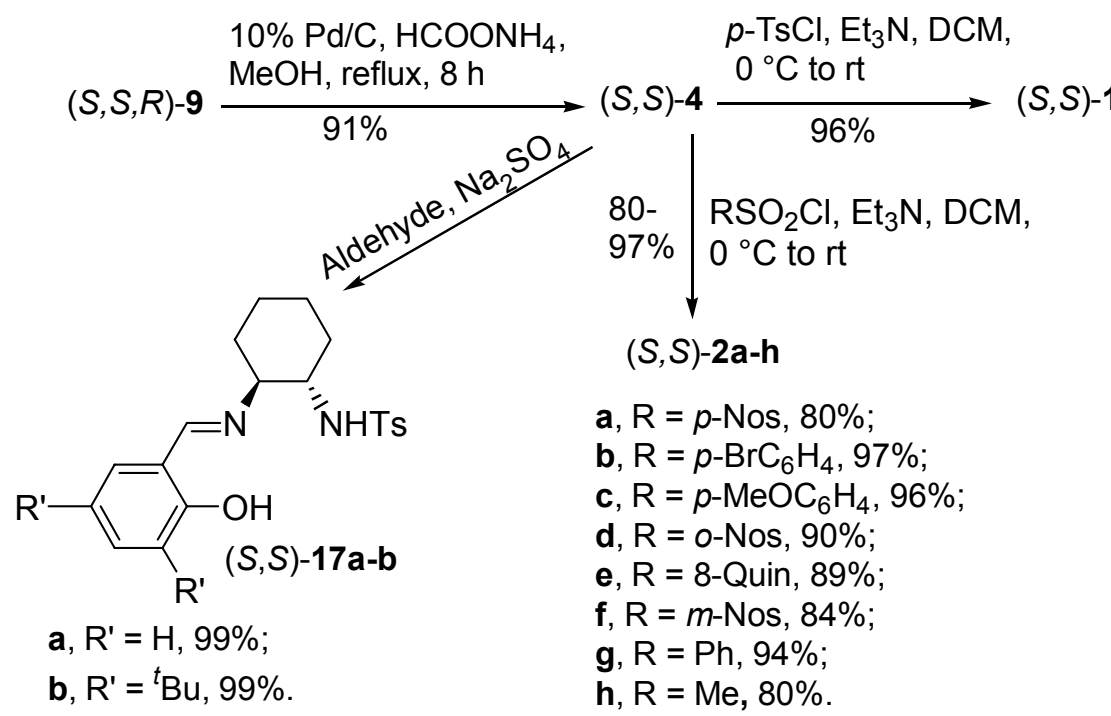

Scheme 2. Synthesis of sulfonamide ligands from an intermediate $(R, R, R)-9$.

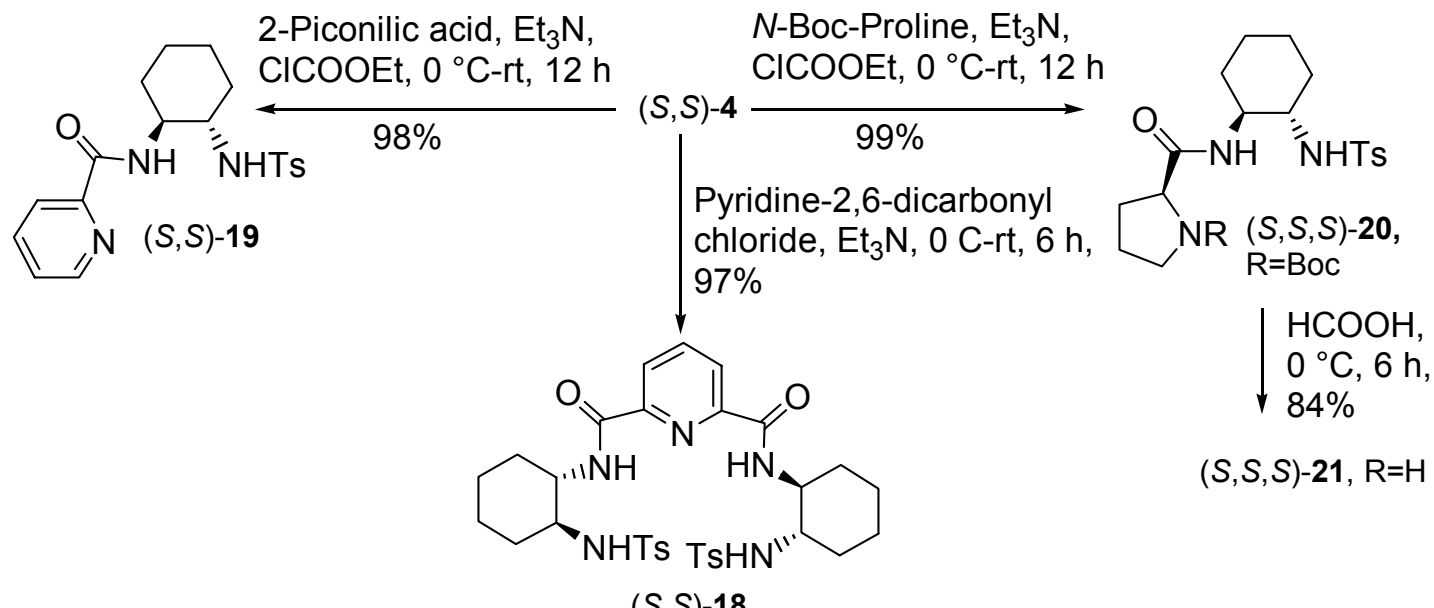

Scheme 3. trans-Cyclohexane-1,2-diamine- based symmetrical and unsymmetrical ligands.

In conclusion, we have developed an efficient method for the desymmetrization of $N$-tosyl aziridines in the presence of $\mathrm{LiClO}_{4}{ }^{22}$ We have used the method to synthesize a variety of symmetrical and unsymmetrical chiral ligands based on trans-1,2-diaminocyclohexane. To the best of our knowledge, this is the first straightforward method for the synthesis of chiral unsymmetrical bis(sulfonamide) ligands based on trans-1,2-diaminocyclohexane. 


\section{Experimental Section}

General Procedures. 1H- NMR spectra were recorded on a $400 \mathrm{MHz}$ spectrometer. Chemical shifts are expressed in ppm downfield from TMS as internal standard, and coupling constants are reported in Hz. Routine monitoring of reaction was performed by TLC, using precoated silica gel TLC plates obtained from E-Merck. All the column chromatographic separations were done by using silica gel (Acme's, 60-120 mesh). The petroleum used was of boiling range $60-80{ }^{\circ} \mathrm{C}$. Reactions that needed anhydrous conditions were run under an atmosphere of nitrogen or argon using flame-dried glassware. The organic extracts were dried over anhydrous sodium sulfate. Evaporation of solvents was performed under reduced pressure. Dichloromethane and acetonitrile were distilled over $\mathrm{CaH}_{2}$, and THF over sodium and benzophenone.

General procedure for ring cleavage of $\mathrm{N}$-tosylaziridine with amine in the presence of LiClO . An amine $(1.25 \mathrm{mmol})$ was added to a solution of $N$-tosylaziridine $(1 \mathrm{mmol})$ and $\mathrm{LiClO}_{4}$ $(0.1 \mathrm{mmol})$ in anhydrous acetonitrile $(6 \mathrm{~mL})$ under argon atmosphere at RT. The reaction mixture was refluxed until completion of the reaction (usually 4-8 h, monitoring by TLC). Most of the acetonitrile was removed in vacuo and the crude reaction mixture was partitioned between EtOAc and water. The organic layer was washed with water, brine, and dried over anhydrous sodium sulfate. It was concentrated in vacuo to give a crude product, which was purified by silica-gel column chromatography using EtOAc and hexane, to give the pure vicinal aminosulfonamide.

N-trans-(2-Benzylamino-cyclohexyl)-4-methyl-benzenesulfonamide (5a). (Table 1, entry 1). Yield 94\%; White solid; mp 118-119 ${ }^{\circ} \mathrm{C} ; R_{\mathrm{f}} 0.38$ (30\% EtOAc in petroleum); IR (film): 3267 , 3022, 2909, 1595, 1329, $1160 \mathrm{~cm}^{-1}$; ${ }^{1} \mathrm{H}-\mathrm{NMR}\left(\mathrm{CDCl}_{3}, 400 \mathrm{MHz}\right) \delta 7.72(\mathrm{~d}, J=8.3 \mathrm{~Hz}, 2 \mathrm{H})$, 7.33-7.18 (m, 7H), $3.67(\mathrm{ABq}, J=78.8 \mathrm{~Hz}, 2 \mathrm{H}), 2.73(\mathrm{~m}, 1 \mathrm{H}), 2.36(\mathrm{~s}, 3 \mathrm{H}), 2.29$ (ddd, $J=10.5$, 10.5, $3.9 \mathrm{~Hz}, 1 \mathrm{H}), 2.05(\mathrm{~m}, 2 \mathrm{H}), 1.65(\mathrm{~m}, 2 \mathrm{H}), 1.12(\mathrm{~m}, 1 \mathrm{H}), 1.18(\mathrm{~m}, 2 \mathrm{H}), 1.01(\mathrm{~m}, 1 \mathrm{H}) ;{ }^{13} \mathrm{C}-$ NMR $\left(\mathrm{CDCl}_{3} 100 \mathrm{MHz}\right) \delta: .143 .11,139.60,137.23,129.49,128.37,127.97,127.07,127.03$, 59.60, 57.21, 49.75, 32.66, 30.90, 24.49, 24.42, 21.43; LCMS (EI, m/z) $358\left(\mathrm{M}^{+}\right)$; Anal. Calcd. for $\mathrm{C}_{20} \mathrm{H}_{26} \mathrm{~N}_{2} \mathrm{O}_{2} \mathrm{~S}$ : C, 67.01; H, 7.31; N, 7.81. Found: C, 67.28; H, 7.72; N, 8.07.

N-trans-[2-(2-Phenylethylamino)-cyclohexyl]-4-methyl-benzenesulfonamide (5b). (Table 1, entry 2). Yield $87 \%$; White solid; mp $129-131^{\circ} \mathrm{C}$ (lit. mp $128{ }^{\circ} \mathrm{C}$ ); $R_{\mathrm{f}} 0.42$ (neat EtOAc); IR (thin film): 2928, 2856, 1598, 1449, $1159 \mathrm{~cm}^{-1} ;{ }^{1} \mathrm{H}-\mathrm{NMR}\left(\mathrm{CDCl}_{3}, 400 \mathrm{MHz}\right) \delta: 7.65(\mathrm{~d}, J=8.0 \mathrm{~Hz}$, 2H), 7.18-7.04 (m, 7H), 2.89-2.85 (m, 1H), 2.71-2.62 (m, 4H), 2.40-2.33 (m, 1H), $2.31(\mathrm{~s}, 3 \mathrm{H})$, $1.95(\mathrm{~m}, 1 \mathrm{H}), 1.79(\mathrm{~m}, 1 \mathrm{H}), 1.59-1.45(\mathrm{M}, 2 \mathrm{H}), 0.78-1.15(\mathrm{~m}, 4 \mathrm{H})$, LCMS (EI, $m / z) 372\left(\mathrm{M}^{+}\right)$; Anal. Calcd. for $\mathrm{C}_{21} \mathrm{H}_{28} \mathrm{~N}_{2} \mathrm{O}_{2} \mathrm{~S}: \mathrm{C}, 67.71 ; \mathrm{H}, 7.58 ; \mathrm{N}, 7.52$. Found: C, 67.40; H, 7.67; N, 7.54.

trans-4-Methyl- $\mathbf{N}$-(2-piperidin-4-yl-cyclohexyl)-benzenesulfonamide (5c). (Table 1, entry 3). Yield 88\%; Colorless gel; $R_{\mathrm{f}} 0.40$ (30\% EtOAc in petroleum); IR (thin film): 3269, 2962, 1598, $1455,1330,1158 \mathrm{~cm}^{-1} ;{ }^{1} \mathrm{H}-\mathrm{NMR}\left(\mathrm{CDCl}_{3}, 400 \mathrm{MHz}\right) \delta: 7.69(\mathrm{~d}, J=8.3,2 \mathrm{H}), 7.22(\mathrm{~d}, J=8.1$ $\mathrm{Hz}, 2 \mathrm{H}), 2.60-2.54(\mathrm{~m}, 1 \mathrm{H}), 2.34(\mathrm{~s}, 3 \mathrm{H}), 2.12-2.05(\mathrm{~m}, 4 \mathrm{H}), 1.72-1.55(\mathrm{~m}, 3 \mathrm{H}), 1.35-0.90(\mathrm{~m}$, 
$11 \mathrm{H})$; LCMS (EI, $m / z) 336\left(\mathrm{M}^{+}\right)$; Anal. Calcd. for $\mathrm{C}_{18} \mathrm{H}_{28} \mathrm{~N}_{2} \mathrm{O}_{2} \mathrm{~S}: \mathrm{C}, 64.25 ; \mathrm{H}, 8.39 ; \mathrm{N}, 8.33$. Found: C, 64.34; H, 8.48; N, 8.35.

trans-4-Methyl- $\mathrm{N}$-(2-morpholin-4-yl-cyclohexyl)-benzenesulfonamide (5d). (Table 1 , entry 4). Yield 95\%; colorless gel; $R_{\mathrm{f}} 0.42$ (30\% EtOAc in petroleum); IR (thin film): 3267, 2960, $1598,1458,1329,1158 \mathrm{~cm}^{-1} ;{ }^{1} \mathrm{H}-\mathrm{NMR}\left(\mathrm{CDCl}_{3}, 400 \mathrm{MHz}\right) \delta: 7.77(\mathrm{~d}, J=8.3,2 \mathrm{H}), 7.30(\mathrm{~d}, J=$ $7.6 \mathrm{~Hz}, 2 \mathrm{H}), 3.49(\mathrm{~m}, 4 \mathrm{H}), 2.70(\mathrm{~m}, 1 \mathrm{H}), 2.45(\mathrm{~m}, 1 \mathrm{H}), 2.42(\mathrm{~s}, 3 \mathrm{H}), 2.12(\mathrm{~m}, 4 \mathrm{H}), 1.75(\mathrm{~m}, 3 \mathrm{H})$, $1.76(\mathrm{~m}, 5 \mathrm{H}) ;{ }^{13} \mathrm{C}-\mathrm{NMR}\left(\mathrm{CDCl}_{3} 100 \mathrm{MHz}\right) \delta: 143.3,136.8,129.6,127.2,67.2,66.8,53.4,53.1$, 31.9, 25.1, 24.1, 22.8, 21.5; LCMS (EI, $m / z) 338\left(\mathrm{M}^{+}\right)$; Anal. Calcd. for $\mathrm{C}_{17} \mathrm{H}_{26} \mathrm{~N}_{2} \mathrm{O}_{3} \mathrm{~S}: \mathrm{C}, 60.33$; H, 7.74; N, 8.28. Found: C, 60.49; H, 7.81; N, 8.31.

trans-4-Methyl- $\boldsymbol{N}$-( $N$ '-ethoxycarbonylpiperizin-4-yl-cyclohexyl)-benzenesulfonamide (5e). (Table 1, entry 5). Yield 96\%; Colorless gel; $R_{\mathrm{f}} 0.41$ (30\% EtOAc in petroleum); ${ }^{1} \mathrm{H}-\mathrm{NMR}$ $\left(\mathrm{CDCl}_{3}, 400 \mathrm{MHz}\right) \delta: 7.66(\mathrm{~d}, J=8.3,2 \mathrm{H}), 7.23(\mathrm{~d}, J=8.0 \mathrm{~Hz}, 2 \mathrm{H}), 3.99(\mathrm{q}, J=7.1 \mathrm{~Hz}, 2 \mathrm{H})$, 3.19 (bs, 4H), 2.62-2.58 (m, 1H), $2.34(\mathrm{~m}, 1 \mathrm{H}), 2.33(\mathrm{~s}, 3 \mathrm{H}), 2.15-2.05(\mathrm{~m}, 5 \mathrm{H}), 1.63-1.57(\mathrm{~m}$, $3 \mathrm{H}), 0.89-1.23(\mathrm{~m}, 7 \mathrm{H})$; LCMS (EI, $m / z) 409\left(\mathrm{M}^{+}\right)$; Anal. Calcd. for $\mathrm{C}_{20} \mathrm{H}_{31} \mathrm{~N}_{3} \mathrm{O}_{4} \mathrm{~S}$ : C, 58.65; $\mathrm{H}$, 7.63; N, 10.23. Found: C, 58.73; H, 7.69; N, 10.21.

N-trans-(2-benzylamino-cyclopentyl)-4-methyl-benzenesulfonamide (6a). (Table 1, entry 6). Yield 88\%; colorless gel; $R_{\mathrm{f}} 0.38$ (30\% EtOAc in petroleum); IR (thin film): 3269, 3029, 2901, 1598, 1330, $1159 \mathrm{~cm}^{-1} ;{ }^{1} \mathrm{H}-\mathrm{NMR}\left(\mathrm{CDCl}_{3}, 400 \mathrm{MHz}\right) \delta: 7.76(\mathrm{~d}, J=8.3 \mathrm{~Hz}, 2 \mathrm{H}), 7.29(\mathrm{~m}, 7 \mathrm{H})$, $3.68(\mathrm{ABq}, J=47.1 \mathrm{~Hz}, 2 \mathrm{H}), 3.26(\mathrm{~m}, 1 \mathrm{H}), 2.83(\mathrm{~m}, 1 \mathrm{H}), 2.39(\mathrm{~s}, 3 \mathrm{H}), 1.91(\mathrm{~m} 2 \mathrm{H}), 1.60(\mathrm{~m}$, 2H), $1.32(\mathrm{~m}, 2 \mathrm{H}) ;{ }^{13} \mathrm{C} \mathrm{NMR}\left(\mathrm{CDCl}_{3} 100 \mathrm{MHz}\right) \delta: 143.4,139.7,137.3,129.7,128.4,128.0$, 127.2, 127.0, 64.4, 59.8, 52.0, 31.2, 29.9, 21.5, 20.7; LCMS (EI, $m / z) 344\left(\mathrm{M}^{+}\right)$; Anal. Calcd. for $\mathrm{C}_{19} \mathrm{H}_{24} \mathrm{~N}_{2} \mathrm{O}_{2} \mathrm{~S}$ : C, 66.25; H, 7.02; N, 8.13. Found: C, 66.32; H, 7.09; N, 8.10.

trans-4-Methyl- $\boldsymbol{N}$-(2-morpholin-4-yl-cyclopentyl)-benzenesulfonamide (6b). (Table 1, entry 7). Yield 93\%; White solid; mp-95-97 ${ }^{\circ} \mathrm{C} ; R_{\mathrm{f}} 0.78$ (30\% EtOAc in petroleum); IR (thin film): 3267, 2960, 1598, 1452, 1329, $1158 \mathrm{~cm}^{-1} ;{ }^{1} \mathrm{H}-\mathrm{NMR}\left(\mathrm{CDCl}_{3}, 400 \mathrm{MHz}\right) \delta: 7.76(\mathrm{~d}, J=8.3 \mathrm{~Hz}$, 2H), 7.30 (d, $J=8.3 \mathrm{~Hz}, 2 \mathrm{H}), 4.85(\mathrm{bs}, 1 \mathrm{H}), 3.54(\mathrm{~m}, 4 \mathrm{H}), 3.23(\mathrm{~m}, 1 \mathrm{H}), 2.52(\mathrm{~m}, 1 \mathrm{H}), 2.38$ (s, $3 \mathrm{H}), 2.34(\mathrm{~m} 2 \mathrm{H}), 2.25(\mathrm{~m}, 2 \mathrm{H}), 1.93(\mathrm{~m}, 1 \mathrm{H}), 1.61(\mathrm{~m}, 3 \mathrm{H}), 1.41(\mathrm{~m}, 2 \mathrm{H}) ;{ }^{13} \mathrm{C}-\mathrm{NMR}\left(\mathrm{CDCl}_{3}\right.$ $100 \mathrm{MHz}) \delta: 143.5,137.0,129.6,127.3,71.9$, 66.9, 55.0, 53.4, 50.2, 31.0, 22.6, 21.5, 20.6; LCMS (EI, $m / z) 324\left(\mathrm{M}^{+}\right)$; Anal. Calcd. for $\mathrm{C}_{16} \mathrm{H}_{24} \mathrm{~N}_{2} \mathrm{O}_{3} \mathrm{~S}: \mathrm{C}, 59.23 ; \mathrm{H}, 7.46$; N, 8.63. Found: C, 59.32; H, 7.54; N, 8.67.

trans- $\boldsymbol{N}$-(6-Benzylamino-cyclohexyl-3-enyl)-4-methyl-benzenesulfonamide (7a). (Table 1, entry 8): Yield 92\%; Colorless gel; $\mathrm{R}_{\mathrm{f}} 0.41$ (30\% EtOAc in petroleum); IR (thin film): 3267, 3028, 2912, 1598, 1331, $1159 \mathrm{~cm}^{-1} ;{ }^{1} \mathrm{H}-\mathrm{NMR}\left(\mathrm{CDCl}_{3}, 400 \mathrm{MHz}\right) \delta: 7.71(\mathrm{~d}, J=8.0 \mathrm{~Hz}, 2 \mathrm{H})$, $7.25(\mathrm{~m}, 7 \mathrm{H}), 5.51(\mathrm{~m}, 2 \mathrm{H}), 3.67(\mathrm{ABq}, J=53.9 \mathrm{~Hz}, 2 \mathrm{H}), 3.04(\mathrm{~m}, 1 \mathrm{H}), 2.65(\mathrm{~m}, 1 \mathrm{H}), 2.51(\mathrm{~m}$, 2H), $2.37(\mathrm{~s}, 3 \mathrm{H}), 2.01(\mathrm{~m}, 1 \mathrm{H}), 1.81(\mathrm{~m}, 1 \mathrm{H}) ;{ }^{13} \mathrm{C}-\mathrm{NMR}\left(\mathrm{CDCl}_{3} 100 \mathrm{MHz}\right) \delta: 143.2,139.7$, 137.0, 129.5, 128.4, 127.9, 127.1, 124.5, 124.4, 55.1, 53.1, 50.2, 32.3, 31.1, 21.4; LCMS (EI, $m / z) 356\left(\mathrm{M}^{+}\right)$; Anal. Calcd. for $\mathrm{C}_{20} \mathrm{H}_{24} \mathrm{~N}_{2} \mathrm{O}_{2} \mathrm{~S}$ : C, 67.38; H, 6.79; N, 7.86. Found: C, 67.51; H, $6.81 ; \mathrm{N}, 7.89$.

trans-4-Methyl- $\boldsymbol{N}$-(6-morpholin-4-yl-cyclohex-3-enyl)-benzenesulfonamide (7b). (Table 1 , entry 9). Yield 89\%; White solid; mp-111-114 ${ }^{\circ} \mathrm{C} ; R_{\mathrm{f}} 0.80$ (30\% EtOAc in petroleum); IR (thin 
film): 3175, 2940, 1598, 1421, 1336, $1162 \mathrm{~cm}^{-1}$; ${ }^{1} \mathrm{H}-\mathrm{NMR}\left(\mathrm{CDCl}_{3}, 400 \mathrm{MHz}\right) \delta: 7.77$ (dd, $J=$ 8.3, $1.7 \mathrm{~Hz}, 2 \mathrm{H}), 7.30(\mathrm{~d}, J=7.6 \mathrm{~Hz}, 2 \mathrm{H}), 3.49(\mathrm{~m}, 4 \mathrm{H}), 2.70(\mathrm{~m}, 1 \mathrm{H}), 2.45(\mathrm{~m}, 1 \mathrm{H}), 2.42(\mathrm{~s}$, $3 \mathrm{H}), 2.12(\mathrm{~m}, 4 \mathrm{H}), 1.75(\mathrm{~m}, 3 \mathrm{H}), 1.76(\mathrm{~m}, 5 \mathrm{H}) ;{ }^{13} \mathrm{C}-\mathrm{NMR}\left(\mathrm{CDCl}_{3} 100 \mathrm{MHz}\right) \delta: 143.3,136.8$, 129.6, 127.2, 67.2, 66.8, 53.4, 53.1, 31.9, 25.1, 24.1, 22.8, 21.5; LCMS (EI, $m / z) 336\left(\mathrm{M}^{+}\right)$; Anal. Calcd. for $\mathrm{C}_{17} \mathrm{H}_{24} \mathrm{~N}_{2} \mathrm{O}_{3} \mathrm{~S}$ : C, 60.69; H, 7.19; N, 8.33. Found: C, 60.83; H, 7.23; N, 8.37.

(1-Benzylamino-2-decanyl)-4-methyl-benzenesulfonamide (8a). (Table 1, entry 10). Yield 89\%; colorless gel; $R_{\mathrm{f}} 0.39$ (30\% EtOAc in petroleum); IR (thin film): 3269, 3027, 2908, 1597 , 1329, $1158 \mathrm{~cm}^{-1}$; ${ }^{1} \mathrm{H}-\mathrm{NMR}\left(\mathrm{CDCl}_{3}, 400 \mathrm{MHz}\right) \delta: 7.64(\mathrm{~d}, J=6.3 \mathrm{~Hz}, 2 \mathrm{H}), 7.62-7.12(\mathrm{~m}, 7 \mathrm{H})$, $3.52(\mathrm{~s}, 2 \mathrm{H}), 3.11-3.14(\mathrm{~m}, 1 \mathrm{H}), 2.31-2.42(\mathrm{~m}, 2 \mathrm{H}), 2.29(\mathrm{~s}, 3 \mathrm{H}), 1.03-1.34(\mathrm{~m}, 18 \mathrm{H}), 0.80(\mathrm{t}, J=$ $6.8 \mathrm{~Hz}, 3 \mathrm{H})$; LCMS (EI, m/z) $444\left(\mathrm{M}^{+}\right)$; Anal. Calcd. for $\mathrm{C}_{26} \mathrm{H}_{40} \mathrm{~N}_{2} \mathrm{O}_{2} \mathrm{~S}: \mathrm{C}, 70.23 ; \mathrm{H}, 9.07$; N, 6.30. Found: C, 70.31; H, 9.09; N, 6.34.

(1-Piperidin-2-decanyl)-4-methyl-benzenesulfonamide (8b). (Table 1, entry 11). Yield 90\%; colorless gel; $R_{\mathrm{f}} 0.41$ (30\% EtOAc in petroleum); IR (thin film): 3269, 1597, 1457, 1331, 1160 $\mathrm{cm}^{-1}$; ${ }^{1} \mathrm{H}-\mathrm{NMR}\left(\mathrm{CDCl}_{3}, 400 \mathrm{MHz}\right) \delta: 7.69(\mathrm{~d}, J=8.3 \mathrm{~Hz}, 2 \mathrm{H}), 7.22(\mathrm{~d}, J=8.3 \mathrm{~Hz}, 2 \mathrm{H}), 3.01-$ $2.94(\mathrm{~m}, 1 \mathrm{H}), 2.34(\mathrm{~s}, 3 \mathrm{H}), 2.23-2.03(\mathrm{~m}, 5 \mathrm{H}), 1.59-1.74(\mathrm{~m}, 1 \mathrm{H}), 1.13-1.34(\mathrm{~m}, 24 \mathrm{H}), 0.84(\mathrm{t}, J$ $=6.4 \mathrm{~Hz}, 3 \mathrm{H})$; LCMS (EI, $\mathrm{m} / z) 422\left(\mathrm{M}^{+}\right)$; Anal. Calcd. for $\mathrm{C}_{24} \mathrm{H}_{42} \mathrm{~N}_{2} \mathrm{O}_{2} \mathrm{~S}: \mathrm{C}, 68.20 ; \mathrm{H}, 10.02 ; \mathrm{N}$, 6.63. Found: C, 68.29; H, 10.09; N, 6.70.

trans-4-Methyl- $N$-[(1S,2S)-2-((R)-1-phenyl-ethylamino)-cyclohexyl]-benzenesulfonamide $((\boldsymbol{S}, \boldsymbol{S}, \boldsymbol{R})-9)$. (Table 2, entry 1): Yield 47\%; white solid; mp-88-90 ${ }^{\circ} \mathrm{C}$; optical rotation $[\alpha]^{25}{ }_{\mathrm{D}}$ +70.28 (c 1.0, $\left.\mathrm{CHCl}_{3}\right) ; R_{\mathrm{f}} 0.28$ (30\% EtOAc in petroleum); IR (thin film): 3271, 2929, 2857, 1599, 1449, 1325, $1162 \mathrm{~cm}^{-1} ;{ }^{1} \mathrm{H}-\mathrm{NMR}\left(\mathrm{CDCl}_{3}, 400 \mathrm{MHz}\right) \delta: 7.69$ (d, $\left.J=8.1 \mathrm{~Hz}, 2 \mathrm{H}\right), 7.25-$ $7.17(\mathrm{~m}, 5 \mathrm{H}), 7.15(\mathrm{~d}, J=8.0 \mathrm{~Hz}, 2 \mathrm{H}), 5.22(\mathrm{bs}, 1 \mathrm{H}, \mathrm{NH}), 3.89(\mathrm{~m}, 1 \mathrm{H}), 2.60(\mathrm{~m}, 1 \mathrm{H}), 2.38(\mathrm{~m}$, $1 \mathrm{H}), 2.29(\mathrm{~s}, 3 \mathrm{H}), 1.95(\mathrm{~m}, 1 \mathrm{H}), 1.85(\mathrm{~m}, 1 \mathrm{H}), 1.49(\mathrm{~m}, 2 \mathrm{H}), 1.26(\mathrm{~d}, J=6.3 \mathrm{~Hz}, 3 \mathrm{H}), 1.89(\mathrm{~m}$, 2H), 1.02 (m, 2H); ${ }^{13} \mathrm{C}-\mathrm{NMR}\left(\mathrm{CDCl}_{3} 100 \mathrm{MHz}\right) \delta: 144.6,143.0,137.5,129.4,128.4,127.3$, 127.0, 126.6, 58.5, 57.6, 55.7, 32.1, 31.6, 24.5, 24.2, 22.6, 21.4; LCMS (EI, $m / z) 372\left(\mathrm{M}^{+}\right)$; Anal. Calcd. for $\mathrm{C}_{21} \mathrm{H}_{28} \mathrm{~N}_{2} \mathrm{O}_{2} \mathrm{~S}$ : C, 67.71; H, 7.58; N, 7.52. Found: C, 67.79; H, 7.63; N, 7.59.

trans-4-Methyl- $N$-[(1R,2R)-2-((R)-1-phenyl-ethylamino)-cyclohexyl]-benzenesulfonamide

$((\boldsymbol{R}, \boldsymbol{R}, \boldsymbol{R})-9)$. (Table 2, entry 1). Yield 47\%; white solid; mp-89-91 ${ }^{\circ} \mathrm{C}$; optical rotation: $[\alpha]^{25}{ }_{\mathrm{D}}$ +6.94 (c 1.0, $\left.\mathrm{CHCl}_{3}\right) ; R_{\mathrm{f}} 0.42$ (30\% EtOAc in petroleum); IR (thin film): 3271, 2930, 2857 , 1601, 1449, 1325, $1161 \mathrm{~cm}^{-1} ;{ }^{1} \mathrm{H}-\mathrm{NMR}\left(\mathrm{CDCl}_{3}, 400 \mathrm{MHz}\right) \delta: 7.75(\mathrm{~d}, J=7.1 \mathrm{~Hz}, 2 \mathrm{H}), 7.25(\mathrm{~m}$, $7 \mathrm{H}), 5.62(\mathrm{bs}, 1 \mathrm{H}), 3.81(\mathrm{~m}, 1 \mathrm{H}), 2.50(\mathrm{~m}, 1 \mathrm{H}), 2.38(\mathrm{~s}, 3 \mathrm{H}), 2.30(\mathrm{~m}, 1 \mathrm{H}), 2.18(\mathrm{~m}, 1 \mathrm{H}), 1.96$ $(\mathrm{m}, 1 \mathrm{H}), 1.61(\mathrm{~m}, 2 \mathrm{H}), 1.25(\mathrm{~d}, J=5.6 \mathrm{~Hz}, 3 \mathrm{H}), 1.16(\mathrm{~m}, 4 \mathrm{H}) ;{ }^{13} \mathrm{C}-\mathrm{NMR}\left(\mathrm{CDCl}_{3} 100 \mathrm{MHz}\right) \delta$ : 146.3, 143.1, 137.1, 129.5, 128.4, 127.2, 127.1, 126.4, 58.2, 58.0, 32.6, 32.0, 24.8, 24.2, 23.2, 21.5; LCMS (EI, $m / z) 372\left(\mathrm{M}^{+}\right)$; Anal. Calcd. for $\mathrm{C}_{21} \mathrm{H}_{28} \mathrm{~N}_{2} \mathrm{O}_{2} \mathrm{~S}: \mathrm{C}, 67.71 ; \mathrm{H}, 7.58$; N, 7.52. Found: C, 67.83; H, 7.65; N, 7.60.

\section{trans-4-Methyl- $N$-[2-((R)-1,2,2-trimethyl-propylamino)-cyclohexyl]-benzenesulfonamide}

(10). (Table 2, entry 2): yield 82\%; colorless gel; diastereomeric ratio $=1: 1$ (determined by ${ }^{1} \mathrm{H}$ NMR); IR (thin film): 3269, 2934, 2861, 1597, 1446, 1328, $1161 \mathrm{~cm}^{-1} ;{ }^{1} \mathrm{H}-\mathrm{NMR}\left(\mathrm{CDCl}_{3}, 400\right.$ $\mathrm{MHz}$ ) for both diastereomers; $\delta: 7.69(\mathrm{~d}, J=8.0 \mathrm{~Hz}, 4 \mathrm{H}), 7.23(\mathrm{~d}, J=8.0 \mathrm{~Hz}, 4 \mathrm{H}), 2.45(\mathrm{~m}, 2 \mathrm{H})$, $2.35(\mathrm{~s}, 3 \mathrm{H}), 2.35(\mathrm{~s}, 3 \mathrm{H}), 2.28-1.95(\mathrm{~m}, 6 \mathrm{H}), 1.59(\mathrm{~m}, 4 \mathrm{H}), 1.15(\mathrm{~m}, 10 \mathrm{H}), 0.87(\mathrm{~d}, J=6.6 \mathrm{~Hz}$, 
3H), $0.81(\mathrm{~m}, 3 \mathrm{H}), 0.80(\mathrm{~s}, 9 \mathrm{H}), 0.71(\mathrm{~s}, 9 \mathrm{H}) ;{ }^{13} \mathrm{C} \mathrm{NMR}\left(\mathrm{CDCl}_{3} 100 \mathrm{MHz}\right)$ for both diastereomers; $\delta$ : 143.0, 137.0, 136.9, 129.4, 129.4, 127.1, 61.8, 60.8, 58.5, 58.0, 57.3, 56.9, 35.2, 33.5, 32.9, 32.6, 31.8, 31.2, 26.5, 26.1, 24.7, 24.3, 24.0, 22.6, 21.4, 21.3, 19.3, 18.1, 14.7; LCMS (EI, $m / z) 352\left(\mathrm{M}^{+}\right)$; Anal. Calcd. for $\mathrm{C}_{19} \mathrm{H}_{32} \mathrm{~N}_{2} \mathrm{O}_{2} \mathrm{~S}: \mathrm{C}, 64.73$; H, 9.15; N, 7.95. Found: C, 64.87; H, 9.19; N, 8.03.

trans-4-Methyl- $N$-[2-((R)-1-phenyl-ethylamino)-cyclopentyl]-benzenesulfonamide

(11).

(Table 2, entry 3). Yield 79\%; colorless gel; diastereomeric ratio $=1: 1$ (determined by ${ }^{1} \mathrm{H}-$

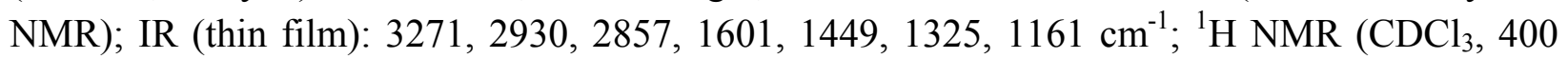
$\mathrm{MHz})$ for both diastereomers; $\delta: 7.68(\mathrm{~m}, 4 \mathrm{H}), 7.16(\mathrm{~m}, 14 \mathrm{H}), 3.75(\mathrm{~m}, 2 \mathrm{H}), 3.20(\mathrm{~m}, 2 \mathrm{H}), 2.75$ $(\mathrm{q}, J=7.6 \mathrm{~Hz}, 1 \mathrm{H}), 2.51(\mathrm{q}, J=8.3 \mathrm{~Hz}, 1 \mathrm{H}), 2.49(\mathrm{~s}, 6 \mathrm{H}), 1.79(\mathrm{~m}, 4 \mathrm{H}), 1.53(\mathrm{~m}, 6 \mathrm{H}), 1.30(\mathrm{~m}$, $1 \mathrm{H}), 1.29(\mathrm{~m}, 6 \mathrm{H}), 1.14(\mathrm{~m}, 1 \mathrm{H}) ;{ }^{13} \mathrm{C}-\mathrm{NMR}\left(\mathrm{CDCl}_{3} 100 \mathrm{MHz}\right)$ for both diastereomers; $\delta$ : 145.6 , 144.6 , 143.3, 143.2, 137.5, 137.3, 129.6, 128.5, 128.3, 127.1, 127.1, 127.0, 126.8, 126.6, 126.3, $63.3,61.9,60.2,59.9,56.9,56.3,30.8,30.7,30.6,29.6,29.3,24.6,23.9,21.4,20.6,20.2$; LCMS (EI, $m / z) 358\left(\mathrm{M}^{+}\right)$; Anal. Calcd. for $\mathrm{C}_{20} \mathrm{H}_{26} \mathrm{~N}_{2} \mathrm{O}_{2} \mathrm{~S}: \mathrm{C}, 67.01$; H, 7.31; N, 7.81. Found: C, 67.21; $\mathrm{H}, 7.40 ; \mathrm{N}, 7.81$.

trans- $\mathrm{N}$-\{2-[(R)-1-(3-Methoxy-phenyl)-ethylamino]-cyclopentyl\}-4-methyl-benzene-

sulfonamide (12). (Table 2, entry 4). Yield 93\%; Colorless gel; diastereomeric ratio = 1:1(determined by $\left.{ }^{1} \mathrm{H} \mathrm{NMR}\right) ;{ }^{1} \mathrm{H}$ - NMR $\left(\mathrm{CDCl}_{3}, 400 \mathrm{MHz}\right)$ for both diastereomers; $\delta: 7.76(\mathrm{~m}$, $4 \mathrm{H})$, 7.28-7.16 (m, 6H), 6.85-6.74 (m, 6H), $3.81(\mathrm{~s}, 3 \mathrm{H}), 3.80(\mathrm{~s}, 3 \mathrm{H}), 3.78(\mathrm{~m}, 1 \mathrm{H}), 3.71(\mathrm{~m}$, $1 \mathrm{H}), 3.21(\mathrm{~m}, 2 \mathrm{H}), 2.74(\mathrm{q}, J=7.6 \mathrm{~Hz}, 1 \mathrm{H}), 2.55(\mathrm{q}, J=8.1 \mathrm{~Hz}, 1 \mathrm{H}), 2.40(\mathrm{~s}, 6 \mathrm{H}), 1.87-1.72(\mathrm{~m}$, $2 \mathrm{H}), 1.63-1.42(\mathrm{~m}, 4 \mathrm{H}), 1.37-1.10(\mathrm{~m}, 12 \mathrm{H}) ;{ }^{13} \mathrm{C}-\mathrm{NMR}\left(\mathrm{CDCl}_{3} 100 \mathrm{MHz}\right)$ for both diastereomers; $\delta$ : 159.7, 159.5, 147.4, 146.6, 143.2, 143.1, 137.5, 137.3, 129.5, 129.4, 129.2, $127.0,127.0,118.9,118.6,112.4,112.2,112.1,111.6,63.3,61.9,60.2,59.8,56.9,56.2,55.1$, 55.0, 30.8, 30.7, 30.5, 29.5, 29.3, 24.6, 23.9, 21.3, 20.6, 20.2; LCMS (EI, $m / z) 388\left(\mathrm{M}^{+}\right.$); Anal. Calcd. for $\mathrm{C}_{21} \mathrm{H}_{28} \mathrm{~N}_{2} \mathrm{O}_{3} \mathrm{~S}$ : C, 64.92; H, 7.26; N, 7.21. Found: C, 65.01; H, 7.29; N, 7.21.

trans-4-Methyl- $N$-[2-((R)-1,2,2-trimethyl-propylamino)-cyclopentyl]-benzenesulfonamide (13). (Table 2, entry 5). Yield 83\%; colorless gel; diastereomeric ratio $=1: 1$ (determined by ${ }^{1} \mathrm{H}-$ NMR); IR (thin film): 3269, 2929, 1598, 1328, $1160 \mathrm{~cm}^{-1} ;{ }^{1} \mathrm{H}-\mathrm{NMR}\left(\mathrm{CDCl}_{3}, 400 \mathrm{MHz}\right)$ for both diastereomers; $\delta: 7.74(\mathrm{~m}, \mathrm{~Hz}, 4 \mathrm{H}), 7.24(\mathrm{~m}, 4 \mathrm{H}), 5.06(\mathrm{bs}, 2 \mathrm{H}), 3.01(\mathrm{~m}, 1 \mathrm{H}), 2.74(\mathrm{~m}, 1 \mathrm{H}), 2.36$ $(\mathrm{s}, 3 \mathrm{H}), 2.05(\mathrm{q}, J=6.4 \mathrm{~Hz}, 1 \mathrm{H}), 1.96(\mathrm{q}, J=6.6 \mathrm{~Hz}, 1 \mathrm{H}), 1.92-1.74(\mathrm{~m}, 4 \mathrm{H}), 1.58-1.47(\mathrm{~m}, 4 \mathrm{H})$, $1.58-1.47(\mathrm{~m}, 4 \mathrm{H}), 1.36-1.29(\mathrm{~m}, 2 \mathrm{H}), 1.14-1.07(\mathrm{~m}, 2 \mathrm{H}), 0.82(\mathrm{~d}, J=6.6 \mathrm{~Hz}, 3 \mathrm{H}), 0.81(\mathrm{~d}, J=$ $6.4 \mathrm{~Hz}, 3 \mathrm{H}), 0.73(\mathrm{~s}, 9 \mathrm{H}) 0.72(\mathrm{~s}, 9 \mathrm{H}) ;{ }^{13} \mathrm{C} \mathrm{NMR}\left(\mathrm{CDCl}_{3} 100 \mathrm{MHz}\right)$ for both diastereomers; $\delta$ : $143.9,143.1,137.5,137.3,129.5,129.5,127.5,127.2,127.1,126.3,64.8,62.5,61.0,60.3,59.9$, 59.5, 46.6, 34.3, 33.9, 31.8, 30.8, 30.5, 29.8, 29.6, 26.8, 26.2, 26.2, 22.6, 21.5, 21.4, 20.9, 20.2, 19.4, 16.0, 14.4; LCMS (EI, $m / z) 388.20\left(\mathrm{M}^{+}\right)$; Anal. Calcd. for $\mathrm{C}_{18} \mathrm{H}_{30} \mathrm{~N}_{2} \mathrm{O}_{2} \mathrm{~S}$ : C, 63.87; H, 8.93; N, 8.28; Found: C, 63.94; H, 9.00; N, 8.29.

trans-4-Methyl- $\boldsymbol{N}$-[6-((R)-1-phenyl-ethylamino)-cyclohex-3-enyl]-benzenesulfonamide (14). (Table 2, entry 6). Yield 83\%; colorless gel; diastereomeric ratio $=1: 1$ (determined by ${ }^{1} \mathrm{H}$ NMR); IR (thin film): 3267, 3027, 2921, 1598, 1449, 1330, $1160 \mathrm{~cm}^{-1} ;{ }^{1} \mathrm{H}-\mathrm{NMR}\left(\mathrm{CDCl}_{3}, 400\right.$ $\mathrm{MHz}$ ) for both diastereomers; $\delta: 7.83-7.71(\mathrm{~m}, 4 \mathrm{H}), 7.34-7.20(\mathrm{~m}, 14 \mathrm{H}), 5.47(\mathrm{~m}, 4 \mathrm{H}), 3.85(\mathrm{~m}$, 
2H), $2.95(\mathrm{~m}, 2 \mathrm{H}), 2.67-2.28(\mathrm{~m}, 6 \mathrm{H}), 2.41(\mathrm{~s}, 3 \mathrm{H}), 2.37(\mathrm{~s}, 3 \mathrm{H}), 2.09-1.56(\mathrm{~m}, 4 \mathrm{H}), 1.29(\mathrm{~d}, J=$ $6.6 \mathrm{~Hz}, 3 \mathrm{H}), 1.25(\mathrm{~d}, J=6.4 \mathrm{~Hz}, 3 \mathrm{H}) ;{ }^{13} \mathrm{C}-\mathrm{NMR}\left(\mathrm{CDCl}_{3} 100 \mathrm{MHz}\right)$ for both diastereomers; $\delta$ : $145.8,144.4,144.1,143.2,143.1,137.2$, 137.0, 129.5, 128.6, 128.4, 127.6, 127.2, 127.1, 126.6, $126.3,124.7,124.4,124.4,124.3,121.5,55.5,54.4,53.5,53.4,52.5,38.5,32.2,32.1,32.0,31.3$, 25.2, 23.3, 23.0, 21.4; LCMS (EI, $m / z) 370\left(\mathrm{M}^{+}\right)$; Anal. Calcd. for $\mathrm{C}_{21} \mathrm{H}_{26} \mathrm{~N}_{2} \mathrm{O}_{2} \mathrm{~S}$ : C, 68.08; $\mathrm{H}$, 7.07; N, 7.56. Found: C, 68.23; H, 7.09; N, 7.61.

General procedure for the synthesis of $(R, R, R)$-2-amino-sulfonamide ligand of trans-1,2diaminocyclohexane. To a vigorously stirred solution of compound $(\boldsymbol{R}, \boldsymbol{R}, \boldsymbol{R})-\mathbf{9}(1 \mathrm{mmol})$ and $\mathrm{K}_{2} \mathrm{CO}_{3}(5 \mathrm{mmol})$ in $\mathrm{MeCN}(6 \mathrm{~mL})$ was added alkyl halide $(3 \mathrm{mmol})$ and the mixture refluxed for 2-24 h. After completion of the reaction the solid residue was filtered off and the filtrate evaporated and directly loaded for silica gel column chromatography to give the pure derivative of 2-amino sulfonamide.

trans-4-Methyl- $N$ - $\{(1 R, 2 R)-2-[$ methyl-((R)-1-phenyl-ethyl)-amino]-cyclohexyl $\}$ -

benzenesulfonamide $((\boldsymbol{R}, \boldsymbol{R}, \boldsymbol{R})-15 \mathrm{a})$ (Scheme 1). (Reagents and conditions: MeI, $\left.70{ }^{\circ} \mathrm{C}, 2 \mathrm{~h}\right)$; Yield $96 \%$; white solid; mp- $112-115^{\circ} \mathrm{C}$; optical rotation: $[\alpha]^{25}{ }_{\mathrm{D}}+28.72$ (c 1.0, $\left.\mathrm{CHCl}_{3}\right) ; R_{\mathrm{f}} 0.58$ (20\% EtOAc in petroleum); IR (KBr pellet): 3172, 2938, 2857, 1598, 1451, 1340, $1162 \mathrm{~cm}^{-1}$; ${ }^{1} \mathrm{H}-$ NMR $\left(\mathrm{CDCl}_{3}, 400 \mathrm{MHz}\right) \delta: 7.23(\mathrm{~d}, J=8.1 \mathrm{~Hz}, 2 \mathrm{H}), 7.36-7.22(\mathrm{~m}, 5 \mathrm{H}), 7.28(\mathrm{~d}, J=8.0 \mathrm{~Hz}$, 2H), $3.56(\mathrm{~m}, 1 \mathrm{H}), 2.66(\mathrm{ddd}, J=10.5,10.3,4.2 \mathrm{~Hz}, 1 \mathrm{H}), 2.41(\mathrm{~s}, 3 \mathrm{H}), 2.19(\mathrm{~s}, 2 \mathrm{H}), 1.74(\mathrm{~s}, 3 \mathrm{H})$, $1.61(\mathrm{~m}, 1 \mathrm{H}), 1.50(\mathrm{~m}, 1 \mathrm{H}), 1.32(\mathrm{~d}, J=6.6 \mathrm{~Hz}, 3 \mathrm{H}), 1.20(\mathrm{~m}, 1 \mathrm{H}), 0.98(\mathrm{~m}, 4 \mathrm{H}) ;{ }^{13} \mathrm{C}-\mathrm{NMR}$ $\left(\mathrm{CDCl}_{3} 100 \mathrm{MHz}\right) \delta: 144.4,143.0,137.2,129.4,128.7,127.3,127.1,61.6,53.8,32.6,31.2$, 29.7, 25.0, 24.1, 22.7, 21.5, 21.3; LCMS (EI, $m / z) 372\left(\mathrm{M}^{+}\right)$; Anal. Calcd. for $\mathrm{C}_{21} \mathrm{H}_{28} \mathrm{~N}_{2} \mathrm{O}_{2} \mathrm{~S}$ : C, 67.71; H, 7.58; N, 7.52. Found: C, 67.85; H, 7.62; N, 7.58.

trans-4-Methyl- $N$ - $\{(1 R, 2 R)-2-[$ ethyl-((R)-1-phenylethyl)amino]cyclohexyl $\}$ benzenesulfonamide ((R,R,R)-15b) (Scheme 1). (Reagents and conditions: EtBr, $\left.70{ }^{\circ} \mathrm{C}, 6 \mathrm{~h}\right)$; yield 92\%; colorless gel; optical rotation $[\alpha]^{25}{ }_{\mathrm{D}}+14.0\left(\mathrm{c} 1.0, \mathrm{CHCl}_{3}\right) ; R_{\mathrm{f}} 0.56$ (20\% EtOAc in petroleum); IR (thin film): 3348, 2933, 2859, 1599, 1449, 1333, $1153 \mathrm{~cm}^{-1} ;{ }^{1} \mathrm{H}-\mathrm{NMR}\left(\mathrm{CDCl}_{3}, 400 \mathrm{MHz}\right) \delta$ : $7.75(\mathrm{~d}, J=8.0 \mathrm{~Hz}, 2 \mathrm{H}), 7.32-7.17(\mathrm{~m}, 7 \mathrm{H}), 3.79(\mathrm{~m}, 1 \mathrm{H}), 3.56(\mathrm{~m}, 1 \mathrm{H}), 3.31-3.28(\mathrm{~m}, 2 \mathrm{H}), 2.56$ (bs, 1H), $2.35(\mathrm{~s}, 3 \mathrm{H}), 1.83(\mathrm{bs}, 1 \mathrm{H}), 1.73(\mathrm{~m}, 1 \mathrm{H}), 1.56(\mathrm{~m}, 1 \mathrm{H}), 1.45(\mathrm{~m}, 2 \mathrm{H}), 1.28(\mathrm{t}, J=7.1$ $\mathrm{Hz}, 3 \mathrm{H}), 1.23(\mathrm{~d}, J=6.4 \mathrm{~Hz}, 3 \mathrm{H}), 1.21(\mathrm{~m}, 1 \mathrm{H}), 0.93(\mathrm{~m}, 2 \mathrm{H}) ;{ }^{13} \mathrm{C}-\mathrm{NMR}\left(\mathrm{CDCl}_{3} 100 \mathrm{MHz}\right) \delta$ : 147.2 , 142.6, 138.3, 129.2, 127.7, 126.6, 126.3, 126.1, 62.5, 57.6, 56.7, 38.2, 33.9, 30.2, 25.3, 24.5, 24.1, 21.1, 16.6; LCMS (EI, m/z) $400\left(\mathrm{M}^{+}\right)$; Anal. Calcd. for $\mathrm{C}_{23} \mathrm{H}_{32} \mathrm{~N}_{2} \mathrm{O}_{2} \mathrm{~S}: \mathrm{C}$, 68.96; $\mathrm{H}$, 8.05; N, 6.99. Found: C, 69.09; H, 8.06; N, 7.01.

\section{trans-4-Methyl- $N$ - $\left\{(1 R, 2 R)-2-\left[{ }^{n}\right.\right.$-propyl-((R)-1-phenyl-ethyl)-amino]-cyclohexyl $\}$ -}

benzenesulfonamide ((R,R,R)-15c) (Scheme 1). (Reagents and conditions: $n$-PrI, $\left.70{ }^{\circ} \mathrm{C}, 14 \mathrm{~h}\right)$; Yield 87\%; colorless gel; optical rotation $[\alpha]_{\mathrm{D}}^{25}+9.6\left(\mathrm{c} 1.0, \mathrm{CHCl}_{3}\right) ; R_{\mathrm{f}} 0.58(20 \%$ EtOAc in petroleum); IR (thin film): 3344, 2930, 2858, 1599, 1450, 1335, $1152 \mathrm{~cm}^{-1} ;{ }^{1} \mathrm{H}-\mathrm{NMR}\left(\mathrm{CDCl}_{3}\right.$, $400 \mathrm{MHz}) \delta: 7.74(\mathrm{~d}, J=8.0 \mathrm{~Hz}, 2 \mathrm{H}), 7.31-7.16(\mathrm{~m}, 7 \mathrm{H}), 3.78-3.76(\mathrm{~m}, 1 \mathrm{H}), 3.48(\mathrm{bs}, 1 \mathrm{H})$, 3.17-3.12 (m, 2H), $2.51(\mathrm{bs}, 1 \mathrm{H}), 2.36(\mathrm{~s}, 3 \mathrm{H}), 1.78-1.67(\mathrm{~m}, 4 \mathrm{H}), 1.59(\mathrm{~m}, 1 \mathrm{H}), 1.45-1.35(\mathrm{~m}$, $2 \mathrm{H}), 1.22(\mathrm{~d}, J=6.6 \mathrm{~Hz}, 3 \mathrm{H}), 1.11-0.96(\mathrm{~m}, 1 \mathrm{H}), 0.93-0.90(\mathrm{t}, J=7.3 \mathrm{~Hz}, 3 \mathrm{H}), 0.85(\mathrm{~m}, 2 \mathrm{H})$; ${ }^{13} \mathrm{C}-\mathrm{NMR}\left(\mathrm{CDCl}_{3} 100 \mathrm{MHz}\right) \delta: 147.3,142.6,138.3,129.3,127.8,126.7,126.3,126.2,62.4$, 
57.7, 56.7, 34.0, 30.3, 25.4, 24.5, 24.3, 24.2, 21.2, 13.9, 11.3; LCMS (EI, $m / z) 414\left(\mathrm{M}^{+}\right)$; Anal. Calcd. for $\mathrm{C}_{24} \mathrm{H}_{34} \mathrm{~N}_{2} \mathrm{O}_{2} \mathrm{~S}: \mathrm{C}, 69.53 ; \mathrm{H}, 8.27 ; \mathrm{N}, 6.76$. Found: C, 69.59; H, 8.30; N, 6.77.

trans-4-Methyl- $N$ - $\left\{(1 R, 2 R)-2-\left[^{n}\right.\right.$-butyl-((R)-1-phenyl-ethyl)-amino]-cyclohexyl $\}$ benzenesulfonamide $((\boldsymbol{R}, \boldsymbol{R}, \boldsymbol{R})-\mathbf{1 5 d})$ (Scheme 1). (Reagents and conditions: $n$-BuBr, $70{ }^{\circ} \mathrm{C}, 24$ h); yield 79\%; colorless gel; optical rotation: $[\alpha]_{\mathrm{D}}^{25}+11.3\left(\mathrm{c} 1.0, \mathrm{CHCl}_{3}\right) ; R_{\mathrm{f}} 0.57(20 \% \mathrm{EtOAc}$ in petroleum); IR (thin film): 3348, 2933, 2860, 1599, 1449, 1335, $1153 \mathrm{~cm}^{-1} ;{ }^{1} \mathrm{H}-\mathrm{NMR}\left(\mathrm{CDCl}_{3}\right.$, $400 \mathrm{MHz}) \delta: 7.74(\mathrm{~d}, J=8.3 \mathrm{~Hz}, 2 \mathrm{H}), 7.30-7.16(\mathrm{~m}, 7 \mathrm{H}), 3.79-3.76(\mathrm{~m}, 1 \mathrm{H}), 3.46$ (bs, 1H), 3.19$3.15(\mathrm{~m}, 2 \mathrm{H}), 2.63(\mathrm{bs}, 1 \mathrm{H}), 2.36(\mathrm{~s}, 3 \mathrm{H}), 1.76-1.56(\mathrm{~m}, 6 \mathrm{H}), 1.48-1.45(\mathrm{~m}, 2 \mathrm{H}), 1.37-1.26(\mathrm{~m}$, 2H), $1.22(\mathrm{~d}, J=6.3 \mathrm{~Hz}, 3 \mathrm{H}), 0.94(\mathrm{t}, J=7.3 \mathrm{~Hz}, 3 \mathrm{H}), 0.85(\mathrm{~m}, 2 \mathrm{H}) ;{ }^{13} \mathrm{C}-\mathrm{NMR}\left(\mathrm{CDCl}_{3} 100\right.$ $\mathrm{MHz}) \delta: 147.4$, 142.7, 138.3, 129.3, 127.9, 126.8, 126.4, 126.3, 62.5, 57.8, 56.7, 43.7, 34.0, 33.3, 30.4, 25.5, 24.5, 24.2, 21.3, 20.2, 13.6; LCMS (EI, $m / z) 428\left(\mathrm{M}^{+}\right)$; Anal. Calcd. for $\mathrm{C}_{25} \mathrm{H}_{36} \mathrm{~N}_{2} \mathrm{O}_{2} \mathrm{~S}$ : C, 70.05; H, 8.47; N, 6.54. Found: C, 70.15; H, 8.51; N, 6.54.

trans-4-Methyl- $N$ - $\{(1 R, 2 R)-2$-[allyl-((R)-1-phenyl-ethyl)-amino]-cyclohexyl $\}$ -

benzenesulfonamide $((\boldsymbol{R}, \boldsymbol{R}, \boldsymbol{R})-\mathbf{1 5 e})$ (Scheme 1). (Reagents and conditions: allyl bromide, $70{ }^{\circ} \mathrm{C}$, $4 \mathrm{~h})$; yield 72\%; colorless gel; optical rotation: $[\alpha]_{\mathrm{D}}^{25}+24.5\left(\mathrm{c} 1.0, \mathrm{CHCl}_{3}\right) ; R_{\mathrm{f}} 0.50(20 \% \mathrm{EtOAc}$ in petroleum); IR (thin film): 3339, 2929, 2857, 1598, 1449, 1335, $1157 \mathrm{~cm}^{-1} ;{ }^{1} \mathrm{H}-\mathrm{NMR}\left(\mathrm{CDCl}_{3}\right.$, $400 \mathrm{MHz}) \delta ; 7.65(\mathrm{~d}, J=8.3 \mathrm{~Hz}, 2 \mathrm{H}), 7.22-7.09(\mathrm{~m}, 7 \mathrm{H}), 5.84-5.77(\mathrm{~m}, 1 \mathrm{H}), 5.16(\mathrm{~d}, J=17.1$ $\mathrm{Hz}, 2 \mathrm{H}), 5.04$ (d, $J=10.0 \mathrm{~Hz}, 2 \mathrm{H}), 3.81$ (d, $J=6.6 \mathrm{~Hz}, 2 \mathrm{H}), 3.68$ (q, $J=6.6 \mathrm{~Hz}, 1 \mathrm{H}), 3.44$ (bs, $1 \mathrm{H}), 2.43(\mathrm{bs}, 1 \mathrm{H}), 2.30(\mathrm{~s}, 3 \mathrm{H}), 1.66-1.36(\mathrm{~m}, 5 \mathrm{H}), 1.13(\mathrm{~d}, J=6.6 \mathrm{~Hz}, 3 \mathrm{H}), 0.90-0.87(\mathrm{~m}, 1 \mathrm{H})$, 0.79-0.76 (m, 2H ); ${ }^{13} \mathrm{C}-\mathrm{NMR}\left(\mathrm{CDCl}_{3} 100 \mathrm{MHz}\right) \delta: 147.5,142.9,138.3,136.1,129.5,128.1$, 127.1, 126.6, 126.4, 117.4, 62.9, 57.3, 56.7, 34.1, 30.8, 25.6, 24.6, 24.5, 21.4; LCMS (EI, $m / z)$ $412\left(\mathrm{M}^{+}\right)$; Anal. Calcd. for $\mathrm{C}_{24} \mathrm{H}_{32} \mathrm{~N}_{2} \mathrm{O}_{2} \mathrm{~S}: \mathrm{C}, 69.87 ; \mathrm{H}, 7.82 ; \mathrm{N}, 6.79$. Found: C, 69.96; H, 7.88; $\mathrm{N}, 6.80$.

\section{trans-4-Methyl- $N$ - $\{(1 R, 2 R)-2-[b e n z y l-((R)-1-p h e n y l-e t h y l)$-amino]-cyclohexyl $\}$ -}

benzenesulfonamide $((\boldsymbol{R}, \boldsymbol{R}, \boldsymbol{R})-\mathbf{1 5 f})$ (Scheme 1). (Reagents and conditions: $\mathrm{PhCH}_{2} \mathrm{Br}, 70{ }^{\circ} \mathrm{C}, 8$ h); yield 78\%; colorless gel; optical rotation: $[\alpha]_{\mathrm{D}}^{25}+30.6\left(\mathrm{c} 1.0, \mathrm{CHCl}_{3}\right) ; R_{\mathrm{f}} 0.54(20 \% \mathrm{EtOAc}$ in petroleum); IR (thin film): 3341, 2926, 2855, 1599, 1449, 1334, $1153 \mathrm{~cm}^{-1} ;{ }^{1} \mathrm{H}-\mathrm{NMR}\left(\mathrm{CDCl}_{3}\right.$, $400 \mathrm{MHz}) \delta: 7.70(\mathrm{~d}, J=8.0 \mathrm{~Hz}, 2 \mathrm{H}), 7.49(\mathrm{~d}, J=7.1 \mathrm{~Hz}, 2 \mathrm{H}), 7.36-7.27(\mathrm{~m}, 3 \mathrm{H}), 7.23-7.11(\mathrm{~m}$, $7 \mathrm{H}), 4.60-4.34(\mathrm{ABq}, J=77.1,15.4 \mathrm{~Hz}, 2 \mathrm{H}), 3.49(\mathrm{~m}, 1 \mathrm{H}), 3.18-3.17(\mathrm{~m}, 1 \mathrm{H}), 2.35(\mathrm{~s}, 3 \mathrm{H})$, $2.34(\mathrm{~m}, 1 \mathrm{H}), 1.57-1.25(\mathrm{~m}, 6 \mathrm{H}), 1.03(\mathrm{~d}, J=6.6 \mathrm{~Hz}, 3 \mathrm{H}), 0.88-0.65(\mathrm{~m}, 2 \mathrm{H}) ;{ }^{13} \mathrm{C}-\mathrm{NMR}\left(\mathrm{CDCl}_{3}\right.$ $100 \mathrm{MHz}) \delta: 147.5,142.9,138.4,138.0,129.5,128.6,128.4,127.9,127.5,127.0,126.5,126.3$, 62.7, 57.6, 56.1, 47.3, 33.8, 30.7, 25.6, 24.3, 24.2, 21.4; LCMS (EI, $m / z) 412\left(\mathrm{M}^{+}\right)$; Anal. Calcd. for $\mathrm{C}_{28} \mathrm{H}_{34} \mathrm{~N}_{2} \mathrm{O}_{2} \mathrm{~S}$ : C, 72.69; H, 7.41; N, 6.06. Found: C, 72.81; H, 7.42; N, 6.09.

Synthesis and characterization of sulfonamide/Schiff base ligand (S,S)-17 (Scheme 2). A solution of $(\boldsymbol{S}, \boldsymbol{S}, \boldsymbol{R})-\mathbf{9}(3 \mathrm{~g}, 8.06 \mathrm{mmol})$ in anhydrous ethanol $(32 \mathrm{~mL})$ was added to a solution of ammonium formate $(5.04 \mathrm{~g}, 80 \mathrm{mmol})$ under argon at RT. To this solution $10 \% \mathrm{Pd}-\mathrm{C}(1 \mathrm{~g})$ was added and refluxed at $80-90{ }^{\circ} \mathrm{C}$ for $8 \mathrm{~h}$. After completion of the reaction the organic solvent was filtered through a Celite bed and concentrated under reduced pressure to give the debenzylated product $(\boldsymbol{S}, \boldsymbol{S})-4$, which was used without purification for the next steps. 
A solution of compound $(\boldsymbol{S}, \boldsymbol{S})-\mathbf{4}(1 \mathrm{mmol})$ and salicylaldehyde $(1 \mathrm{mmol})$ in $\mathrm{MeOH}(10 \mathrm{~mL})$ was treated with $\mathrm{Na}_{2} \mathrm{SO}_{4}(500 \mathrm{mg})$ and the reaction mixture was left at RT overnight. After completion of the reaction (checked by running TLC), the solid residue was filtered off and the filtrate concentrated. The crude material was purified by column chromatography over silica gel to give the pure sulfonamide/Schiff base ligand $(S, S)-17$; Yield $99 \%$; yellow solid; mp $68-70^{\circ} \mathrm{C}$; $[\alpha]_{\mathrm{D}}^{25}-41.2$ (c 1.0, $\left.\mathrm{CHCl}_{3}\right) ; R_{\mathrm{f}} 0.44$ (30\% EtOAc in petroleum); IR (KBr pellet): 3292, 2955, 2863, 1628, 1443, 1323, 1159, $1090 \mathrm{~cm}^{-1}$; ${ }^{1} \mathrm{H}-\mathrm{NMR}\left(\mathrm{CDCl}_{3}, 400 \mathrm{MHz}\right) \delta: 8.24(\mathrm{~s}, 1 \mathrm{H}), 7.62$ (d, $J=8.3 \mathrm{~Hz}, 2 \mathrm{H}), 7.37$ (d, $J=2.2 \mathrm{~Hz}, 1 \mathrm{H}), 7.09$ (d, $J=8.0 \mathrm{~Hz}, 2 \mathrm{H}), 6.97(\mathrm{~d}, J=2.4 \mathrm{~Hz}, 1 \mathrm{H}), 4.68$ $(\mathrm{d}, J=5.8 \mathrm{~Hz}, 1 \mathrm{H}), 3.20(\mathrm{~m}, 1 \mathrm{H}), 2.97(\mathrm{~m}, 1 \mathrm{H}), 2.33(\mathrm{~m}, 1 \mathrm{H}), 2.26(\mathrm{~s}, 3 \mathrm{H}), 1.74(\mathrm{~m}, 3 \mathrm{H}), 1.42(\mathrm{~s}$, 9H), 1.29-1.33 (m, 4H), $1.26(\mathrm{~s}, 9 \mathrm{H}) ;{ }^{13} \mathrm{C}-\mathrm{NMR}\left(\mathrm{CDCl}_{3} 100 \mathrm{MHz}\right) \delta: 166.7,157.7,142.9,140.1$, 137.1, 136.3, 129.5, 127.2, 126.8, 126.1, 117.5, 71.8, 57.5, 34.9, 34.0, 33.6, 32.7, 31.5, 29.4, 24.2, 23.7, 21.5; Anal. Calcd. for $\mathrm{C}_{28} \mathrm{H}_{40} \mathrm{~N}_{2} \mathrm{O}_{3} \mathrm{~S}$ : C, 69.38; H, 8.32; N, 5.78. Found: C, 69.72; H, 8.44; N, 5.79.

Synthesis and characterization of mixed bis-sulfonamide ligands of trans-1,2cyclohexanediamine (Scheme 2). To a stirred solution of compound $(\boldsymbol{S}, \boldsymbol{S})-\mathbf{4}(1 \mathrm{mmol})$ and triethylamine $(3 \mathrm{mmol})$ in dry $\mathrm{CH}_{2} \mathrm{Cl}_{2}(6 \mathrm{~mL})$ was added the aryl- or alkyl- sulfonyl chloride $(1.1$ $\mathrm{mmol})$ at $0{ }^{\circ} \mathrm{C}$. The reaction mixture was gradually warmed to $\mathrm{RT}$ and then left for $6-8 \mathrm{~h}$. After completion of the reaction (checked by TLC), it was diluted with $6 \mathrm{~mL}$ of $\mathrm{CH}_{2} \mathrm{Cl}_{2}$ and washed with $2 \mathrm{~N} \mathrm{HCl}$, water, and brine. The organic layer was dried over anhydrous $\mathrm{Na}_{2} \mathrm{SO}_{4}$ and the solvent was evaporated. The crude material was purified by column chromatography over silica gel to get the pure mixed bis-sulfonamide ligands of trans-1,2-cyclohexanediamine.

(S,S)-2a. Yield 80\%; pale yellowish solid; mp-215-217 ${ }^{\circ} \mathrm{C} ;[\alpha]^{25}+5.4\left(\mathrm{c} 1.0, \mathrm{CHCl}_{3}\right) ; R_{\mathrm{f}} 0.60$ (50\% EtOAc in petroleum); IR (KBr pellet): 3257, 2932, 2858, 1531, 1435, $1350 \mathrm{~cm}^{-1}$; ${ }^{1} \mathrm{H}-\mathrm{NMR}$ $\left(\mathrm{CDCl}_{3}, 400 \mathrm{MHz}\right) \delta: 8.37(\mathrm{~d}, J=7.1 \mathrm{~Hz}, 2 \mathrm{H}), 8.13(\mathrm{~d}, J=6.8 \mathrm{~Hz}, 2 \mathrm{H}), 7.68(\mathrm{~d}, J=8.3 \mathrm{~Hz}, 2 \mathrm{H})$, $7.31(\mathrm{~d}, J=8.3 \mathrm{~Hz}, 2 \mathrm{H}), 5.73(\mathrm{~d}, J=4.6 \mathrm{~Hz}, 1 \mathrm{H}, \mathrm{NH}), 4.39(\mathrm{~d}, J=7.4 \mathrm{~Hz}, 1 \mathrm{H}, \mathrm{NH}), 2.78(\mathrm{~m}$, $2 \mathrm{H}), 2.43(\mathrm{~s}, 3 \mathrm{H}), 2.1-5-2.13(\mathrm{~m}, 1 \mathrm{H}), 1.65-1.42(\mathrm{~m}, 3 \mathrm{H}), 0.86-1.33(\mathrm{~m}, 4 \mathrm{H}) ;{ }^{13} \mathrm{C}-\mathrm{NMR}\left(\mathrm{CDCl}_{3}\right.$ $100 \mathrm{MHz}) \delta: 149.8,145.7,143.8,136.6,129.8,128.5,126.7,124.2,57.7,55.8,33.9,32.7,24.3$, 23.8, 21.4; MS (FAB) $454\left(\mathrm{M}^{+}+1\right)$; Anal. Calcd. for $\mathrm{C}_{19} \mathrm{H}_{23} \mathrm{~N}_{3} \mathrm{O}_{6} \mathrm{~S}_{2}$ : C, 50.32; $\mathrm{H}, 5.11$; N, 9.27. Found: C, 50.43; H, 5.15; N, 9.27.

(S,S)-2b. Yield 97\%; white solid; mp-175-177 ${ }^{\circ} \mathrm{C} ;[\alpha]^{25}$ - -8.6 (c 1.0, $\left.\mathrm{CHCl}_{3}\right) ; R_{\mathrm{f}} 0.42(30 \%$ EtOAc in Petroleum ether); IR (thin film): 3282, 2937, 2860, 1327, $1159 \mathrm{~cm}^{-1} ;{ }^{1} \mathrm{H} \mathrm{NMR}\left(\mathrm{CDCl}_{3}\right.$, $400 \mathrm{MHz}) \delta: 7.78(\mathrm{~d}, J=8.6 \mathrm{~Hz}, 2 \mathrm{H}), 7.73(\mathrm{~d}, J=8.3 \mathrm{~Hz}, 2 \mathrm{H}), 7.66(\mathrm{~d}, J=8.6 \mathrm{~Hz}, 2 \mathrm{H}), 7.31$ (d, $J=8.0 \mathrm{~Hz}, 2 \mathrm{H}), 5.46(\mathrm{~d}, J=4.6 \mathrm{~Hz}, 1 \mathrm{H}, \mathrm{NH}), 4.91(\mathrm{~d}, J=6.3 \mathrm{~Hz}, 1 \mathrm{H}, \mathrm{NH}), 2.80$ (bs, 2H), 2.42 $(\mathrm{s}, 3 \mathrm{H}), 2.16-1.91(\mathrm{~m}, 1 \mathrm{H}), 1.68-1.55(\mathrm{~m}, 2 \mathrm{H}), 0.93-1.09(\mathrm{~m}, 5 \mathrm{H}) ;{ }^{13} \mathrm{C} \mathrm{NMR}\left(\mathrm{CDCl}_{3} 100 \mathrm{MHz}\right)$ $\delta: 143.7,139.2,136.9,132.3,129.8,128.8,127.5,127.0,57.0,56.2,33.3,32.7,24.2,23.9,21.5$; MS (FAB) $487\left(\mathrm{M}^{+}\right), 489\left(\mathrm{M}^{+}+2\right)$; Anal. Calcd. for $\mathrm{C}_{19} \mathrm{H}_{23} \mathrm{BrN}_{2} \mathrm{O}_{4} \mathrm{~S}_{2}: \mathrm{C}, 46.82 ; \mathrm{H}, 4.76 ; \mathrm{N}$, 5.75. Found: C, 46.91; H, 4.79; N, 5.75.

$(\boldsymbol{S}, \boldsymbol{S})$-2c. Yield $96 \%$; white solid; mp- $135-137^{\circ} \mathrm{C}$; $[\alpha]_{\mathrm{D}}^{25}-7.0$ (c $\left.1.0, \mathrm{CHCl}_{3}\right) ; R_{\mathrm{f}} 0.40(50 \%$ EtOAc in petroleum); IR (KBr pellet): 3291, 2940, 2869, 1597, 1420, 1324, $1158 \mathrm{~cm}^{-1}$; ${ }^{1} \mathrm{H}-$ $\operatorname{NMR}\left(\mathrm{CDCl}_{3}, 400 \mathrm{MHz}\right) \delta: 7.81(\mathrm{~d}, J=8.8 \mathrm{~Hz}, 2 \mathrm{H}), 7.75(\mathrm{~d}, J=8.3 \mathrm{~Hz}, 2 \mathrm{H}), 7.31(\mathrm{~d}, J=8.3$ 
$\mathrm{Hz}, 2 \mathrm{H}), 6.78$ (d, $J=8.3 \mathrm{~Hz}, 2 \mathrm{H}), 5.13(\mathrm{~d}, J=5.6 \mathrm{~Hz}, 1 \mathrm{H}, \mathrm{NH}), 5.09$ (d, $J=5.6 \mathrm{~Hz}, 1 \mathrm{H}, \mathrm{NH})$, $3.86(\mathrm{~s}, 3 \mathrm{H}), 2.75(\mathrm{bs}, 2 \mathrm{H}), 2.42(\mathrm{~s}, 3 \mathrm{H}), 1.99-2.00(\mathrm{~m}, 1 \mathrm{H}), 1.81-1.79(\mathrm{~m}, 2 \mathrm{H}), 1.54-1.53(\mathrm{~m}$, 2H), 1.15-1.09 (m, 3H); ${ }^{13} \mathrm{C}-\mathrm{NMR}\left(\mathrm{CDCl}_{3} 100 \mathrm{MHz}\right) \delta: 162.8,143.5,136.9$, 131.4, 129.7, 129.3, 127.1 , 114.2, 56.4, 55.5, 33.0, 29.6, 24.0, 21.5; MS (FAB) $439\left(\mathrm{M}^{+}+1\right)$; Anal. Calcd. for $\mathrm{C}_{20} \mathrm{H}_{26} \mathrm{~N}_{2} \mathrm{O}_{5} \mathrm{~S}_{2}$ : C, 54.77; H, 5.98; N, 6.39. Found: C, 54.89; H, 5.97; N, 6.40.

$(\boldsymbol{S}, \boldsymbol{S})-2 d$. Yield $90 \%$; pale yellowish solid; mp-89-91 ${ }^{\circ} \mathrm{C} ;[\alpha]^{25}-12.87\left(\mathrm{c} 1.0, \mathrm{CHCl}_{3}\right) ; R_{\mathrm{f}} 0.60$ (50\% EtOAc in petroleum); IR (thin film): 3297, 2937, 2861, 2360, 1596, $1163 \mathrm{~cm}^{-1}$; ${ }^{1} \mathrm{H}-\mathrm{NMR}$ $\left(\mathrm{CDCl}_{3}, 400 \mathrm{MHz}\right) \delta: 8.14(\mathrm{dd}, J=7.2,2.4 \mathrm{~Hz}, 1 \mathrm{H}), 7.87-7.56(\mathrm{~m}, 3 \mathrm{H}), 7.73(\mathrm{~d}, J=8.8 \mathrm{~Hz}$, 2H), $7.30(\mathrm{~d}, J=8.4 \mathrm{~Hz}, 2 \mathrm{H}), 5.43(\mathrm{~d}, J=6.8 \mathrm{~Hz}, 1 \mathrm{H}, \mathrm{NH}), 5.10(\mathrm{~d}, J=6.8 \mathrm{~Hz}, 1 \mathrm{H}, \mathrm{NH}), 3.17-$ $3.13(\mathrm{~m}, 1 \mathrm{H}), 2.90-2.86(\mathrm{~m}, 1 \mathrm{H}), 2.42(\mathrm{~s}, 3 \mathrm{H}), 1.89-1.81(\mathrm{~m}, 2 \mathrm{H}), 1.57-1.56(\mathrm{~m}, 2 \mathrm{H}), 1.24-1.13$ $(\mathrm{m}, 4 \mathrm{H}) ;{ }^{13} \mathrm{C}-\mathrm{NMR}\left(\mathrm{CDCl}_{3} 100 \mathrm{MHz}\right) \delta: 147.6,143.5,137.0,134.0,133.7,133.0,130.7,129.7$, 129.5, 128.2, 127.0, 125.3, 60.4, 57.2, 56.5, 33.0, 24.0, 23.8, 21.5; MS (FAB) $454\left(\mathrm{M}^{+}+1\right)$; Anal. Calcd. for $\mathrm{C}_{19} \mathrm{H}_{23} \mathrm{~N}_{3} \mathrm{O}_{6} \mathrm{~S}_{2}$ : C, 50.32; H, 5.11; N, 9.27. Found: C, 50.45; H, 5.12; N, 9.29.

(S,S)-2e. Yield 89\%; White solid; mp-96-99 ${ }^{\circ} \mathrm{C} ;[\alpha]^{25}$ - $32.3\left(\right.$ c $\left.1.0, \mathrm{CHCl}_{3}\right) ; R_{\mathrm{f}} 0.29(50 \%$ EtOAc in petroleum); IR (thin film): 3270, 2929, 2857, 2359, 2338, 1429, $1162 \mathrm{~cm}^{-1} ;{ }^{1} \mathrm{H}-\mathrm{NMR}\left(\mathrm{CDCl}_{3}\right.$, $400 \mathrm{MHz}) \delta: 9.01(\mathrm{dd}, J=4.1,1.7 \mathrm{~Hz}, 1 \mathrm{H}), 8.42(\mathrm{dd}, J=7.3,1.4 \mathrm{~Hz}, 1 \mathrm{H}), 8.30(\mathrm{dd}, J=8.6,1.7$ $\mathrm{Hz}, 1 \mathrm{H}), 8.09$ (dd, $J=8.1,1.2 \mathrm{~Hz}, 1 \mathrm{H}), 7.76(\mathrm{~d}, J=8.3 \mathrm{~Hz}, 2 \mathrm{H}), 7.67$ (t, $J=7.6 \mathrm{~Hz}, 1 \mathrm{H}), 7.58-$ $7.55(\mathrm{~m}, 1 \mathrm{H}), 7.29(\mathrm{~d}, J=8.3 \mathrm{~Hz}, 2 \mathrm{H}), 5.87(\mathrm{~d}, J=6.3 \mathrm{~Hz}, 1 \mathrm{H}), 5.49-5.48(\mathrm{~m}, 1 \mathrm{H}), 2.97-2.94(\mathrm{~m}$, $1 \mathrm{H}), 2.81-2.76(\mathrm{~m}, 1 \mathrm{H}), 2.42(\mathrm{~s}, 1 \mathrm{H}), 2.15-2.12(\mathrm{~m}, 1 \mathrm{H}), 1.65(\mathrm{bs}, 1 \mathrm{H}), 0.94-1.52(\mathrm{~m}, 6 \mathrm{H}) ;{ }^{13} \mathrm{C}-$ NMR $\left(\mathrm{CDCl}_{3} 100 \mathrm{MHz}\right) \delta: 151.4,143.1,143.0,137.0,136.0,133.6,131.0,129.6,128.9,127.4$, 126.9, 125.7, 122.4, 57.3, 56.4, 33.5, 32.3, 24.2, 23.7, 21.6; MS (FAB) $460\left(\mathrm{M}^{+}+1\right)$; Anal. Calcd. for $\mathrm{C}_{22} \mathrm{H}_{25} \mathrm{~N}_{3} \mathrm{O}_{4} \mathrm{~S}_{2}$ : C, 57.49; H, 5.48; N, 9.14. Found: C, 57.62; H, 5.49; N, 9.15.

$(\boldsymbol{S}, \boldsymbol{S})-2 f$. Yield $84 \%$; yellow solid; mp-185-187 ${ }^{\circ} \mathrm{C} ;[\alpha]_{\mathrm{D}}^{25}-12.9$ (c $\left.1.0, \mathrm{CHCl}_{3}\right) ; R_{\mathrm{f}} 0.39(40 \%$ EtOAc in petroleum); IR (KBr pellet): 3315, 3222, 2937, 2864, 1607, 1535, 1325, $1158 \mathrm{~cm}^{-1}$; ${ }^{1} \mathrm{H}-\mathrm{NMR}\left(\mathrm{CDCl}_{3}, 400 \mathrm{MHz}\right) \delta: 8.73(\mathrm{~s}, 1 \mathrm{H}), 8.42(\mathrm{~d}, J=8.0 \mathrm{~Hz}, 1 \mathrm{H}), 8.29(\mathrm{~d}, J=7.6 \mathrm{~Hz}, 1 \mathrm{H})$, $7.79(\mathrm{t}, J=8.1 \mathrm{~Hz}, 1 \mathrm{H}), 7.66(\mathrm{~d}, J=8.0 \mathrm{~Hz}, 2 \mathrm{H}), 7.29(\mathrm{~d}, J=8.0 \mathrm{~Hz}, 2 \mathrm{H}), 5.77(\mathrm{~d}, J=5.6 \mathrm{~Hz}$, $1 \mathrm{H}), 4.71(\mathrm{~d}, J=7.8 \mathrm{~Hz}, 1 \mathrm{H}), 2.89-2.77(\mathrm{~m}, 2 \mathrm{H}), 2.42(\mathrm{~s}, 3 \mathrm{H}), 2.11-2.04(\mathrm{~m}, 1 \mathrm{H}), 1.62-1.47(\mathrm{~m}$, $3 \mathrm{H}), 1.18-1.01(\mathrm{~m}, 4 \mathrm{H}) ;{ }^{13} \mathrm{C}-\mathrm{NMR}\left(\mathrm{CDCl}_{3} 100 \mathrm{MHz}\right) \delta: 148.1,143.8,142.4,136.9,133.1$, 130.7, 129.8, 127.0, 122.4, 60.4, 57.9, 56.0, 34.0, 32.6, 23.9, 21.5; MS (FAB) $454\left(\mathrm{M}^{+}+1\right)$; Anal. Calcd. for $\mathrm{C}_{19} \mathrm{H}_{23} \mathrm{~N}_{3} \mathrm{O}_{6} \mathrm{~S}_{2}$ : C, 50.32; H, 5.11; N, 9.27. Found: C, 50.47; H, 5.12; N, 9.29.

$(\boldsymbol{S}, \boldsymbol{S})-2 g$. Yield 94\%; White solid; mp-110-112 ${ }^{\circ} \mathrm{C} ;[\alpha]^{25}-15.3$ (c 1.0, $\left.\mathrm{CHCl}_{3}\right) ; R_{\mathrm{f}} 0.59(40 \%$ EtOAc in Petroleum ether); IR (KBr pallet): 3283, 2932, 2869, 1420, 1324, $1162 \mathrm{~cm}^{-1}$; ${ }^{1} \mathrm{H}$ NMR $\left(\mathrm{CDCl}_{3}, 400 \mathrm{MHz}\right) \delta: 7.90-7.86(\mathrm{~m}, 2 \mathrm{H}), 7.74(\mathrm{~d}, J=8.8 \mathrm{~Hz}, 2 \mathrm{H}), 7.60-7.51(\mathrm{~m}, 3 \mathrm{H}), 7.31(\mathrm{~d}, J$ $=8.0 \mathrm{~Hz}, 2 \mathrm{H}), 5.22(\mathrm{~d}, J=6.0 \mathrm{~Hz}, 1 \mathrm{H}, \mathrm{NH}), 5.01(\mathrm{~d}, J=6.4 \mathrm{~Hz}, 1 \mathrm{H}, \mathrm{NH}), 2.78(\mathrm{bs}, 2 \mathrm{H}), 2.42(\mathrm{~s}$, $3 \mathrm{H}), 1.87-1.74(\mathrm{~m}, 2 \mathrm{H}), 1.54-1.53(\mathrm{~m}, 2 \mathrm{H}), 0.97-1.08(\mathrm{~m}, 4 \mathrm{H}) ;{ }^{13} \mathrm{C} \mathrm{NMR}\left(\mathrm{CDCl}_{3} 100 \mathrm{MHz}\right) \delta$ : 143.5, 139.9, 136.9, 132.7, 129.7, 129.1, 127.1, 127.0, 56.7, 56.4, 33.2, 32.9, 24.1, 24.0, 21.5; MS (FAB) $409\left(\mathrm{M}^{+}+1\right)$; Anal. Calcd. for $\mathrm{C}_{19} \mathrm{H}_{24} \mathrm{~N}_{2} \mathrm{O}_{4} \mathrm{~S}_{2}$ : C, 55.86; H, 5.92; N, 6.86. Found: C, 55.99; H, 5.97; N, 6.86.

$(\boldsymbol{S}, \boldsymbol{S})$-2h. Yield 80\%; White solid; mp-98-101 ${ }^{\circ} \mathrm{C} ;[\alpha]^{25}-45.3$ (c 1.0, $\left.\mathrm{CHCl}_{3}\right) ; R_{\mathrm{f}} 0.41(60 \%$ EtOAc in petroleum); IR (KBr pallet): 3284, 2939, 2860, 1446, 1320, 1158, $1091 \mathrm{~cm}^{-1} ;{ }^{1} \mathrm{H}-$ 
NMR $\left(\mathrm{CDCl}_{3}, 400 \mathrm{MHz}\right) \delta: 7.76(\mathrm{~d}, J=8.3 \mathrm{~Hz}, 2 \mathrm{H}), 7.32(\mathrm{~d}, J=8.6 \mathrm{~Hz}, 2 \mathrm{H}), 5.09(\mathrm{~d}, J=7.6$ $\mathrm{Hz}, 1 \mathrm{H}, \mathrm{NH}), 4.85$ (d, $J=6.8 \mathrm{~Hz}, 1 \mathrm{H}, \mathrm{NH}), 3.22(\mathrm{~m}, 1 \mathrm{H}), 3.05(\mathrm{~s}, 3 \mathrm{H}), 2.98-2.79(\mathrm{~m}, 1 \mathrm{H}), 2.43$ $(\mathrm{s}, 3 \mathrm{H}), 2.17-2.15(\mathrm{~m}, 1 \mathrm{H}), 1.71-1.60(\mathrm{~m}, 4 \mathrm{H}), 1.33-1.05(\mathrm{~m}, 3 \mathrm{H}) ;{ }^{13} \mathrm{C}-\mathrm{NMR}\left(\mathrm{CDCl}_{3} 100 \mathrm{MHz}\right)$ $\delta: 143.7,137.3,129.9,127.0,57.6,56.9 ; 41.5,24.5,24.3,21.5$; MS (FAB) $347\left(\mathrm{M}^{+}+1\right)$; Anal. Calcd. for $\mathrm{C}_{14} \mathrm{H}_{22} \mathrm{~N}_{2} \mathrm{O}_{4} \mathrm{~S}_{2}$ : C, 48.53; H, 6.40; N, 8.09. Found: C, 48.69; H, 6.41; N, 8.10.

Synthesis of $C_{2}$-symmetric pyridyl bis(amide) bis(sulfonamide) ligand $(S, S)-18$. 2,6Pyridinedicarbonyl dichloride $(1.1 \mathrm{mmol})$ was slowly added to a solution of $(S, S)-4(1 \mathrm{mmol})$ and triethylamine $(5 \mathrm{mmol})$ in dichloromethane $(5 \mathrm{~mL})$ at $0{ }^{\circ} \mathrm{C}$. The mixture was stirred at RT for $6 \mathrm{~h}$. The resulting solution was washed with water, then brine, and dried. Solvent removal and purification over silica gel by column chromatography gave the pure product $(S, S)$-18; yield 97\%; white solid; mp-158- $160^{\circ} \mathrm{C} ;[\alpha]^{25}{ }_{\mathrm{D}}-38.3$ (c $\left.1.0, \mathrm{CHCl}_{3}\right) ; R_{\mathrm{f}} 0.40(80 \% \mathrm{EtOAc}$ in petroleum); IR (KBr pellet): 3344, 2933, 2861, 1667, 1538, 1447, 1322, 1156, 1090, $1007 \mathrm{~cm}^{-1}$; ${ }^{1} \mathrm{H} \mathrm{NMR}\left(\mathrm{CDCl}_{3}, 400 \mathrm{MHz}\right) \delta: 8.55(\mathrm{~d}, J=7.8 \mathrm{~Hz}, 2 \mathrm{H}), 8.20(\mathrm{~d}, J=7.8 \mathrm{~Hz}, 2 \mathrm{H}), 7.95(\mathrm{t}, J=7.8$ $\mathrm{Hz}, 1 \mathrm{H}), 7.71(\mathrm{~d}, J=8.0 \mathrm{~Hz}, 4 \mathrm{H}), 7.04(\mathrm{~d}, J=8.1 \mathrm{~Hz}, 4 \mathrm{H}), 5.61(\mathrm{~d}, J=8.3 \mathrm{~Hz}, 2 \mathrm{H}), 3.78-3.80$ (m, 2H), 3.33-3.35 (m, 2H), $2.23(\mathrm{~s}, 6 \mathrm{H}), 2.19-2.20(\mathrm{~m}, 2 \mathrm{H}), 1.81-1.87(\mathrm{~m}, 3 \mathrm{H}), 1.63-1.66(\mathrm{~m}$, $4 \mathrm{H}), 1.09-1.47(\mathrm{~m}, 7 \mathrm{H}) ;{ }^{13} \mathrm{C}-\mathrm{NMR}\left(\mathrm{CDCl}_{3} 100 \mathrm{MHz}\right) \delta: 164.1,148.3,142.8,138.4,138.3$, 129.4, 126.5, 124.4, 57.3, 53.7, 34.1, 31.9, 25.1, 24.3, 21.4; Anal. Calcd. for $\mathrm{C}_{33} \mathrm{H}_{41} \mathrm{~N}_{5} \mathrm{O}_{6} \mathrm{~S}_{2}$ : C, 59.35; H, 6.19; N, 10.49. Found: C, 59.66; H, 6.26; N, 10.47.

Synthesis of pyridyl amide sulfonamide ligand $(S, S)-19$. A solution of 2-picolinic acid (1 mmol) and triethylamine $(3 \mathrm{mmol})$ in anhydrous THF $(5 \mathrm{~mL})$ was treated with ethyl chloroformate $(1.1 \mathrm{mmol})$ at $0{ }^{\circ} \mathrm{C}$ for $30 \mathrm{~min}$. A solution of $(\boldsymbol{S}, \boldsymbol{S})-\mathbf{4}(1 \mathrm{mmol})$ in THF $(5 \mathrm{~mL})$ was added dropwise and the reaction mixture was left at RT overnight. After completion of the reaction (checked by TLC), most of the THF was removed in vacuo and the crude reaction mixture was partitioned between EtOAc and water. The organic layer was washed with water, brine, and dried over anhydrous sodium sulfate. It was concentrated in vacuo to give a crude product, which was purified by silica gel column chromatography using EtOAc and hexane to give pure $(\boldsymbol{S}, \boldsymbol{S})-\mathbf{1 9}$; Yield 98\%; White solid; mp $180-182^{\circ} \mathrm{C} ;[\alpha]^{25}-1.2$ (c 1.0, $\left.\mathrm{CHCl}_{3}\right) ; R_{\mathrm{f}} 0.40$ (50\% EtOAc in petroleum); IR (KBr pallet): 3319, 3062, 2937, 2859, 1657, 1594, 1516, 1443, 1318, 1154, 1084, $1000 \mathrm{~cm}^{-1} ;{ }^{1} \mathrm{H}-\mathrm{NMR}\left(\mathrm{CDCl}_{3}, 400 \mathrm{MHz}\right) \delta: 8.42(\mathrm{~d}, J=4.9 \mathrm{~Hz}, 1 \mathrm{H}), 8.11(\mathrm{~d}$, $J=7.6 \mathrm{~Hz}, 1 \mathrm{H}), 7.88(\mathrm{dt}, J=15.4,7.8,1.4 \mathrm{~Hz}, 1 \mathrm{H}), 7.65(\mathrm{~d}, J=8.0 \mathrm{~Hz}, 2 \mathrm{H}), 7.53(\mathrm{~d}, J=8.0 \mathrm{~Hz}$, 2H), $7.45(\mathrm{~m}, 1 \mathrm{H}), 6.75(\mathrm{~d}, J=8.1 \mathrm{~Hz}, 2 \mathrm{H}), 6.01(\mathrm{~d}, J=5.6 \mathrm{~Hz}, 1 \mathrm{H}), 3.76-3.84(\mathrm{~m}, 1 \mathrm{H}), 2.96-$ $3.04(\mathrm{~m}, 1 \mathrm{H}), 2.22-2.26(\mathrm{~m}, 1 \mathrm{H}), 2.12(\mathrm{~s}, 3 \mathrm{H}), 1.95-1.98(\mathrm{~m}, 1 \mathrm{H}), 1.73-1.78(\mathrm{~m}, 3 \mathrm{H}), 1.25-1.51$ $(\mathrm{m}, 3 \mathrm{H}) ;{ }^{13} \mathrm{C}-\mathrm{NMR}\left(\mathrm{CDCl}_{3} 100 \mathrm{MHz}\right) \delta: 165.4,148.8,147.7,142.1,137.8,137.3,129.1,126.5$, 126.2, 122.3, 60.2, 51.9, 34.9, 32.2, 24.5, 24.4, 21.3; Anal. Calcd. for $\mathrm{C}_{19} \mathrm{H}_{23} \mathrm{~N}_{3} \mathrm{O}_{3} \mathrm{~S}$ : C, 61.10; $\mathrm{H}$, 6.21; N, 11.25; Found: C, 61.34; H, 6.32; N, 11.29.

$(\boldsymbol{S}, \mathbf{S}, \boldsymbol{S})-20$. The procedure was same as the synthesis of $(\boldsymbol{S}, \boldsymbol{S})-19$ except $N$-Boc-L-Proline was used instead of 2-Picolinic acid; Yield 99\%; White solid; mp-184-186 ${ }^{\circ} \mathrm{C}$; $[\alpha]^{25} \mathrm{D}-74.0$ (c 1.0, $\left.\mathrm{CHCl}_{3}\right) ; R_{\mathrm{f}} 0.45$ (60\% EtOAc in Petroleum ether); IR (KBr pallet): 3310, 2934, 2866, 1668, 1534, 1393, 1327, 1250, 1162, 1091, $912 \mathrm{~cm}^{-1} ;{ }^{1} \mathrm{H}$ NMR $\left(\mathrm{CDCl}_{3}, 400 \mathrm{MHz}\right) \delta: 7.69(\mathrm{~d}, J=8.0$ $\mathrm{Hz}, 2 \mathrm{H}), 7.25$ (br, 2H), 7.20 (br, 1H, NH), 6.03 (bs, 1H, NH), 3.69 (d, J=7.8 Hz, 2H), 3.47 (br, 
1H), $3.32(\mathrm{~m}, 1 \mathrm{H}), 3.21(\mathrm{~m}, 1 \mathrm{H}), 2.92(\mathrm{~m}, 1 \mathrm{H}), 2.41(\mathrm{~s}, 3 \mathrm{H}), 2.30$ (br, 1H), 1.67-2.17 (m, 7H), 1.43 (s, 9H), 1.19-1.33 (m, 4H); Anal. Calcd. for $\mathrm{C}_{23} \mathrm{H}_{35} \mathrm{~N}_{3} \mathrm{O}_{5} \mathrm{~S}$ : C, 59.33; H, 7.58; N, 9.02; Found: C, 59.61; H, 7.62; N, 9.09.

$(\boldsymbol{R}, \boldsymbol{R}, \boldsymbol{S})-\mathbf{2 0}$. The procedure was the same as for the synthesis of $(\boldsymbol{S}, \mathbf{S}, \boldsymbol{S})-\mathbf{2 0}$ except that amino sulfonamide $(\boldsymbol{R}, \boldsymbol{R})-4$ was used; yield $90 \%$; white solid; mp-186-188 ${ }^{\circ}$; $[\alpha]^{25}-27.5$ (c 1.0 , $\left.\mathrm{CHCl}_{3}\right) ; R_{\mathrm{f}} 0.65\left(80 \%\right.$ EtOAc in petroleum); ${ }^{1} \mathrm{H}-\mathrm{NMR}\left(\mathrm{CDCl}_{3}, 400 \mathrm{MHz}\right) \delta: 7.72(\mathrm{~d}, J=8.3$ $\mathrm{Hz}, 2 \mathrm{H}), 7.27(\mathrm{~d}, J=8.1 \mathrm{~Hz}, 2 \mathrm{H}), 6.52(\mathrm{br}, 1 \mathrm{H}), 4.24(\mathrm{bs}, 1 \mathrm{H}), 3.56-3.66(\mathrm{~m}, 2 \mathrm{H}), 3.03-(\mathrm{br}, 1 \mathrm{H})$, 2.41 (s, 3H), 2.21 (br, 1H), 2.05 (br, 2H), 1.84 (m, 1H), 1.59-1.67 (m, 5H), 1.46 (s, 9H), 1.161.27 (m, 4H); Anal. Calcd. for $\mathrm{C}_{23} \mathrm{H}_{35} \mathrm{~N}_{3} \mathrm{O}_{5} \mathrm{~S}$ : C, 59.33; H, 7.58; N, 9.02; Found: C, 59.59; H, $7.63 ; \mathrm{N}, 9.09$.

Synthesis of (2S, 1'S, 2'S)-pyrrolidine-2-carboxylic acid [2'-(4-methylphenylsulfonamido)cyclohexyl]-amide $(\boldsymbol{S}, \mathbf{S}, \boldsymbol{S})$-21. The $N$-Boc protected amido sulfonamide was added portionwise to a chilled solution of formic acid at $0{ }^{\circ} \mathrm{C}$ and reaction mixture was stirred at the same temperature for 6-8 h. After completion of the reaction (checked by TLC), most of the $\mathrm{HCOOH}$ was removed in vacuo and the crude reaction mixture was basified with $25 \%$ ammonia solution. It was partitioned between EtOAc and water. The organic layer was washed with water, brine, and dried over anhydrous sodium sulfate. It was concentrated in vacuo to give a crude product, which was purified by silica gel column chromatography using EtOAc and hexane to give pure $(\boldsymbol{S}, \mathbf{S}, \boldsymbol{S})$-21. Yield 84\%; white solid; mp- $168-170^{\circ} \mathrm{C} ;[\alpha]^{25} \mathrm{D}-29.5$ (c $\left.1.0, \mathrm{CHCl}_{3}\right) ; R_{\mathrm{f}} 0.30(5 \%$ $\mathrm{MeOH}$ in $\mathrm{CH}_{2} \mathrm{Cl}_{2}$ ); IR (KBr pellet): 3366, 3287, 3123, 2956, 2862, 1634, 1529, 1454, 1320, 1161, 1100, 914, $810 \mathrm{~cm}^{-1} ;{ }^{1} \mathrm{H}-\mathrm{NMR}\left(\mathrm{CDCl}_{3}, 400 \mathrm{MHz}\right) \delta: 7.62$ (br, 2H), 7.53 (d, J=8.8 Hz, 2H), 7.16 (br, 2H), 6.13 (bs, $1 \mathrm{H}), 3.54$ (dd, $J=8.8,6.1 \mathrm{~Hz}, 1 \mathrm{H}), 3.48$ (br, $1 \mathrm{H}), 2.78-2.87$ (m, $3 \mathrm{H}), 2.28(\mathrm{~s}, 3 \mathrm{H}), 1.99-2.02(\mathrm{~m}, 1 \mathrm{H}), 1.57-1.91(\mathrm{~m}, 7 \mathrm{H}), 1.10-1.21(\mathrm{~m}, 4 \mathrm{H}) ;{ }^{13} \mathrm{C}-\mathrm{NMR}\left(\mathrm{CDCl}_{3}\right.$ $100 \mathrm{MHz}) \delta: 175.0,142.3,138.6,129.1,126.4,59.7,58.3,51.7,46.4,33.2,31.9,30.1$, 25.3, 24.3, 24.1, 21.0; Anal. Calcd. for $\mathrm{C}_{18} \mathrm{H}_{27} \mathrm{~N}_{3} \mathrm{O}_{3} \mathrm{~S}$ : C, 59.15; H, 7.45; N, 11.50. Found: C, 59.30; H, $7.53 ; \mathrm{N}, 11.51$.

Synthesis of (2S, 1'R, 2'R)-pyrrolidine-2-carboxylic acid [2'-(4-methylphenylsulfonamido)cyclohexyl]-amide $(\boldsymbol{R}, \boldsymbol{R}, \boldsymbol{S})-21$. Yield $90 \%$; white crystalline solid; mp-172-174 ${ }^{\circ} \mathrm{C} ;[\alpha]^{25}{ }_{\mathrm{D}}+10.4$ (c $\left.1.0, \mathrm{CHCl}_{3}\right) ; R_{\mathrm{f}} 0.25\left(5 \% \mathrm{MeOH}\right.$ in $\left.\mathrm{CH}_{2} \mathrm{Cl}_{2}\right) ;{ }^{1} \mathrm{H}-\mathrm{NMR}\left(\mathrm{CDCl}_{3}, 400 \mathrm{MHz}\right) \delta: 7.88(\mathrm{~d}, J=8.3$ $\mathrm{Hz}, 1 \mathrm{H}), 7.56$ (m, 2H), 7.09 (m, 2H), 6.29 (bs, 1H), 3.68-3.72 (m, 1H), 3.44 (bs, 1H), 2.82-2.91 $(\mathrm{m}, 3 \mathrm{H}), 2.23(\mathrm{~s}, 3 \mathrm{H}), 1.87-2.00(\mathrm{~m}, 1 \mathrm{H}), 1.42-1.85(\mathrm{~m}, 7 \mathrm{H}), 1.04-1.10(\mathrm{~m}, 4 \mathrm{H}) ;{ }^{13} \mathrm{C}-\mathrm{NMR}$ $\left(\mathrm{CDCl}_{3} 100 \mathrm{MHz}\right) \delta: 174.2,142.9,139.3,129.7,126.7,60.2,57.7,52.6,46.9,32.7,32.4,30.7$, 25.9, 24.8, 23.7, 21.5; Anal. Calcd. for $\mathrm{C}_{18} \mathrm{H}_{27} \mathrm{~N}_{3} \mathrm{O}_{3} \mathrm{~S}$ : C, 59.15; H, 7.45; N, 11.50. Found: C, 59.37; H, 7.49; N, 11.63 .

\section{Acknowledgements}


We thank the Department of Science and Technology, Government of India for a research grant. A.B. thanks Council of Scientific and Industrial Research, New Delhi for a Senior Research Fellowship.

\section{References and Notes}

1. (a) Ojima, I., Ed. Catalytic Asymmetric Synthesis; $2^{\text {nd }}$ Edn, Wiley: New York, 2000. (b) Noyori, R. Asymmetric Catalysis in Organic Synthesis; Wiley: New York, 1994.

2. Reviews: (a) Pfaltz, A. Chimia 2004, 58, 49. (b) Schoffers, E. Eur. J. Org. Chem. 2003, 7, 1145. (c) Johnson, J. S.; Evans, D. A. Acc. Chem. Res. 2000, 33, 325. (d) Helmchen, G.; Pfaltz, A. Acc. Chem. Res. 2000, 33, 336. (e) Hayashi, T. Acc. Chem. Res. 2000, 33, 354. (f) Jacobsen, E. N. Acc. Chem. Res. 2000, 33, 421-431. (g) Ghosh, A. K.; Mathivanan, P.; Cappiello, J. Tetrahedron: Asymmetry 1998, 9, 1.

3. (a) Kaellstroem, K.; Hedberg, C.; Brandt, P.; Bayer, A.; Andersson, P.G. J. Am. Chem. Soc. 2004, 126, 14308. (b) Vidal-Ferran, A.; Moyano, A.; Pericas, M. A.; Riera, A. J. Org. Chem. 1997, 62, 4970.

4. (a) Pritchett, S.; Woodmansee, D. H.; Gantzel, P.; Walsh, P. J. J. Am. Chem. Soc. 1998, 120, 6423. (b) Pritchett, S.; Gantzel, P.; Walsh, P. J. Organometallics 1997, 16, 5130. (c) Armistead, L. T.; White, P. S.; Gagne, M. R. Organometallics 1998, 17, 216.

5. Corey, E. J.; Sarshar, S.; Lee, D.-H. J. Am. Chem. Soc. 1994, 116, 12089.

6. Corey, E. J.; Letaric, M. A. J. Am. Chem. Soc. 1995, 117, 9616.

7. (a) Lutz, C.; Knochel, P. J. Org. Chem. 1997, 62, 7895. (b) Vettel, S.; Lutz, C.; Diefenbach, A.; Harderlein, G.; Hammerschmidt, S.; Kühling, K.; Mofid, M. R.; Zimmermann, T.; Knochel, P. Tetrahedron: Asymmetry 1997, 8, 779. (c) Pritchett, S.; Woodmansee, D. H.; Davis, T. J.; Walsh, P. J. Tetrahedron Lett. 1998, 39, 5941. (d) Halm, C.; Kurth, M. J. Angew. Chem., Int. Ed. 1998, 37, 510.

8. (a) Denmark, S. E.; Christenson, B. L.; Coe, D. M.; O’Connor, S. P. Tetrahedron Lett. 1995, 36, 2215. (b) Denmark, S. E.; Christenson, B. L.; O’Connor, S. P. Tetrahedron Lett. 1995, 36, 2219. (c) Denmark, S. E.; O’Connor, S. P. J. Org. Chem. 1997, 62, 3390. (d) Denmark, S. E.; O’Connor, S. P.; Wilson, S. R. Angew. Chem., Int. Ed. 1998, 37, 1149.

9. (a) Evans, D. A.; Nelson, S. G. J. Am. Chem. Soc. 1997, 119, 6452. (b) For the use of $\mathrm{C}_{2-}$ symmetric trans-1,2-cyclohexanediamine based ligands in other enantioselective reactions, see: Mastranzo, V. M.; Quintero, L.; de Parrodi, C. A.; Juaristi, E.; Walsh, P. J. Tetrahedron 2004, 60, 1781. (c) de Parrodi, C. A.; Walsh, P. J. Synlett 2004, 2417.

10. (a) For asymmetric cyclopropanation using sulfonamide/Schiff base chiral ligands, see: Balsells, J.; Walsh, P. J. J. Org. Chem. 2000, 65, 5005-5008. (b) For asymmetric transfer hydrogenation of ketones using chiral amino-sulfonamide, see: Ptintener, K.; Schwink, L.; Knochel, P. Tetrahedron Lett. 1996, 37, 8165. (c) For the synthesis and application of some other hybrid ligands of chiral 1,2-diaminocyclohexane, see: Kim, Y. K.; Lee, S. J.; Ahn, K. H. J. Org. Chem. 2000, 65, 7807. 
11. For application of other kind of chiral amino-sulfonamide ligands in asymmetric synthesis, see: (a) Xue, D.; Chen, Y.-C.; Cui, X.; Wang, Q.-W.; Zhu, J.; Deng, J.-G. J. Org. Chem. 2005, 70, 3584. (b) Duncan, A. P.; Leighton, J. L. Org. Lett. 2004, 6, 4117. (c) For application of unsymmetrical bis(sulfonamide)ligand, see: Balsells, J.; Betancort, J. M.; Walsh, P. J. Angew. Chem. Int. Ed. Engl. 2000, 39, 3428.

12. Frost, C. G.; Mendonca, P. Tetrahedron: Asymmetry. 1999, 10, 1831.

13. (a) Balsells, J.; Mejorado, L.; Phillips, M.; Ortega, F.; Aguirre, G.; Somanathan, R.; Walsh, P. J. Tetrahedron: Asymmetry 1998, 9, 4135. (b) For improved procedure for the synthesis of 4, see: Ng, K.; Somanathan, R.; Walsh, P. J. Tetrahedron: Asymmetry 2001, 12, 1719. (c) For a preliminary account of this work, see: Bisai, A.; Prasad, B. A. B.; Singh, V. K. Tetrahedron Lett. 2005, 46, 7935.

14. (a) Jeong, J. U; Tao, B.; Sagasser, I.; Henniges, H.; Sharpless, K. B. J. Am. Chem. Soc. 1998, 120, 6844. (b) Vyas, R.; Gao, G-Y.; Harden, J. D.; Zhang, X. P. Org. Lett. 2004, 6, 1907. (c) Thakur, V. V.; Sudalai, A. Tetrahedron Lett. 2003, 44, 989. (d) Dauban, P.; Saniere, L.; Tarrade, A.; Dodd, R. H. J. Am. Chem. Soc. 2001, 123, 7707.

15. (a) Fan, R-H.; Hou, X-L. J. Org. Chem. 2003, 68, 726. (b) Hou, X-L.; Fan, R-H.; Dai, L-X. J. Org. Chem. 2002, 67, 5295. (c) Yadav, J. S.; Reddy, B. V. S.; Jyothirmai, B.; Murthy, M. S. R. Synlett 2002, 1, 53. (d) Sekar, G, Singh, V. K. J. Org. Chem. 1999, 64, 2537. (e)For the synthesis of trans-1,2-cyclohexanediamine derivatives through the ring cleavage of cyclohexyl- $N$-alkylaziridine, see: de Parrodi, C. A.; Moreno, G. E.; Quintero, L.; Juaristi, E. Tetrahedron: Asymmetry 1998, 9, 2093. (f) de Parrodi, C. A.; Vazquez, V.; Quintero, L.; Juaristi, E. Synth. Commun. 2001, 31, 3295.

16. (a) Watson, I. D. G.; Yudin, A. K. J. Org. Chem. 2003, 68, 5160. (b) Cossy, J; Bellosta, V; Alauze, V; Desmurs, J-R. Synthesis 2002, 15, 2211. (c) Lake, F.; Moberg, C. Eur. J. Org. Chem. 2002, 3179. (d) Paul, B. J.; Hobbs, E.; Buccino, P.; Hudlicky, T. Tetrahedron Lett. 2001, 42, 6433. (e) Meguro, M.; Asao, N.; Yamamoto, Y. Tetrahedron Lett. 1994, 35, 7395.

17. Chakraborty, T. K.; Ghosh, A.; Raju, T. V. Chem. Lett. 2003, 32, 82.

18. See experimental section.

19. Crystallographic data for $(R, R, R)-\mathbf{1 5 a}$ and $(R, R, R)-\mathbf{1 6}$ have been deposited with the Cambridge Crystallographic Data Centre as supplementary publications no. CCDC-280469 and 280470, respectively. This data can be obtained free of charge via the internet http://www.ccdc.cam.ac.uk/conts/retrieving.html or by sending an email to deposit@ccdc.cam.ac.uk.

20. Ray, M.; Ghosh, D.; Shirin, Z.; Mukherjee, R. Inorg. Chem. 1997, 36, 3568.

21. Chen, J.-R.; Lu, H.-H.; Li, X.-Y.; Cheng, L.; Wan, J.; Xiao, W.-J. Org. Lett. 2005, 7, 4543.

22. Although $\mathrm{LiClO}_{4}$ is potentially explosive, the chance of an explosion in $\mathrm{MeCN}$ is less when used in catalytic amount. 